\title{
Análisis y perspectivas del estudio de los ecosistemas terrestres de México: dinámica hidrológica y flujos de nitrógeno y fósforo
}

\author{
Analysis and perspectives of the study of terrestrial ecosystems in Mexico: hydrological dynamics \\ and fluxes of nitrogen and phosphorus
}

\author{
Angelina Martínez-Yrízar ${ }^{\mathrm{a}, *}$, Javier Álvarez-Sánchez ${ }^{\mathrm{b}}$ y Manuel Maass ${ }^{\mathrm{c}}$ \\ ${ }^{a}$ Instituto de Ecología, Universidad Nacional Autónoma de México, Colosio y Sahuaripa s/n, Los Arcos, 83250 Hermosillo, Sonora, México \\ ${ }^{\mathrm{b}}$ Facultad de Ciencias, Universidad Nacional Autónoma de México, Circuito Exterior Universitario $5 / n$, Cd. Universitaria, 04510 Ciudad de México, México \\ ${ }^{\mathrm{c}}$ Instituto de Investigaciones en Ecosistemas y Sustentabilidad (IIES), Universidad Nacional Autónoma de México, Apartado postal 27-3, Santa María de Guido, \\ 58090 Morelia, Michoacán, México \\ Recibido el 28 de septiembre de 2016; aceptado el 31 de julio de 2017 \\ Disponible en Internet el 28 de noviembre de 2017
}

\section{Resumen}

Presentamos un diagnóstico del estado actual de conocimiento en México sobre 2 grandes temas de la ecología de ecosistemas: la dinámica hidrológica y los flujos de nitrógeno y fósforo de los ecosistemas terrestres. Una revisión de la literatura en revistas indizadas (1990 a 2016) arrojó 100 publicaciones, aproximadamente la mitad de cada tema, de 135 autores nacionales y 63 del extranjero, la mayoría en la última década. Un grupo reducido de sitios representativos de matorrales xerófilos, bosques tropicales caducifolios, bosques templados y bosques de niebla han sido intensamente estudiados. Son escasos los trabajos con un enfoque integral, y pocos estudios exploran las interacciones agua-nutrientes. La mayoría de los trabajos son de corto plazo ( $<5$ años) y a escala espacial pequeña (parcela, ladera o cuenca). La incorporación de metodologías más sofisticadas (isótopos estables, sensoría remota, torres «Eddy Covariance») y el trabajo en redes nacionales han permitido fortalecer la investigación ecosistémica en México. Gradualmente se han mejorado las condiciones para abordar vacíos de información y las oportunidades de incidir en la toma de decisiones sobre la gestión del agua, protección de hábitats críticos para la provisión de servicios ambientales, la conservación y el manejo de ecosistemas.

(C) 2017 Universidad Nacional Autónoma de México, Instituto de Biología. Este es un artículo Open Access bajo la licencia CC BY-NC-ND (http://creativecommons.org/licenses/by-nc-nd/4.0/).

Palabras clave: Ecohidrología; Partición de la precipitación; Humedad del suelo; Evapotranspiración; Escorrentía; Flujos; Nutrientes

\section{Abstract}

Here we assess the current state of the art of two major research fields in ecosystem ecology in Mexico: hydrological dynamics and fluxes of nitrogen and phosphorus of terrestrial ecosystems. This assessment was based on a review of published indexed papers (1990 to 2016). We found 100 papers, approximately half in hydrology and half in biogeochemistry, authored by 135 Mexican and 63 international scientists. Most of these studies were published in the last decade. A small group of field sites from desert scrub, tropical dry forests and temperate and cloud forests have been intensively investigated. Research with an integrated approach are scarce (most studies analyzed one or a few components of the hydrology or nutrient cycling in relation to abiotic factors) and fewer investigated water-nutrient interactions. Most of the studies were short-term $(<5$ years $)$

\footnotetext{
* Autor para correspondencia.

Correo electrónico: angelina@unam.mx (A. Martínez-Yrízar).

La revisión por pares es responsabilidad de la Universidad Nacional Autónoma de México.
} 
and were conducted at the plot, slope or watershed spatial level. New methodologies (stable isotopes, remote sensing, Eddy Covariance towers) and collaborative research networks have substantially advanced ecosystem research in Mexico. Gradually, the ability to investigate research gaps of ecosystem function have improved the opportunities to inform decisions regarding water management, protection of critical habitats for the provision of ecosystem services, conservation and ecosystem management.

(C) 2017 Universidad Nacional Autónoma de México, Instituto de Biología. This is an open access article under the CC BY-NC-ND license (http://creativecommons.org/licenses/by-nc-nd/4.0/).

Keywords: Ecohydrology; Precipitation partition; Soil water content; Evapotranspiration; Runoff; Fluxes; Nutrients

\section{Introducción}

La ecología como disciplina científica dentro de la biología surge del análisis de la relación entre los organismos y su ambiente. Inicialmente dominó un enfoque de investigación en los individuos, poblaciones y comunidades, centrándose en estudiar y describir los mecanismos que han permitido el proceso evolutivo de las especies, incluyendo los factores que determinan la distribución y la abundancia de sus poblaciones y la estructura y dinámica de las comunidades bióticas que constituyen; lo que denominamos genéricamente como la «historia natural» del mundo biológico. Con el surgimiento de la teoría de sistemas a comienzos del siglo pasado, y su incorporación en la ecología varias décadas después, se comenzó a estudiar al fenómeno biológico a escalas por encima del nivel organísmico, identificando a los ecosistemas como la conformación de sistemas complejos integrados por agentes bióticos y abióticos interactuando en un espacio y tiempo determinados. En contraste con la ecología organísmica, la ecología de ecosistemas centra su atención en los procesos funcionales del ecosistema a diferentes escalas de tiempo y espacio, mediante el análisis de sus almacenes, balances y flujos de agua, energía y nutrientes. Conforme se ha ido reconociendo la crisis ambiental global (Millennium Ecosystem Assessment, 2005) resulta imprescindible entender procesos ecológicos por encima del nivel organísmico, por lo que el dominio de la perspectiva analítico-reduccionista de la biología de los organismos ha ido cediendo el paso a un enfoque más sistémico y funcional de los ecosistemas a escalas local, regional y global.

Las investigaciones en el campo de la ecología de ecosistemas, con un enfoque funcional de los ecosistemas terrestres de México, iniciaron formalmente a principios de la década de 1980 con estudios cuantitativos, principalmente sobre productividad primaria y ciclos de nutrientes en bosques tropicales y templados. La presencia de estaciones de campo en algunas de las áreas naturales protegidas de México fue un detonador importante para impulsar la investigación ecológica con un enfoque ecosistémico en el país. Inicialmente dominaron los estudios de carácter descriptivo. La innovación y la adopción de nuevas tecnologías (p.ej., isotopía estable, percepción remota, el uso de torres «Eddy Covariance», registradores automáticos de datos en tiempo real), enfoques filogenéticos y análisis de metadatos, entre otros, han permitido diseñar estudios que integran variables ambientales y biológicas a diferentes escalas y analizar las interacciones, logrando con ello una mejor aproximación para identificar los mecanismos y factores que regulan la dinámica del agua y los flujos de nutrientes en los ecosistemas.
La investigación ecológica con un enfoque ecosistémico es por naturaleza multidisciplinaria y colectiva, por lo que el surgimiento de redes y programas multi-institucionales ha sido un elemento necesario y a la vez detonador de esta investigación en México (Maass, Díaz-Delgado, Balvanera, Castillo y Martínez-Yrízar, 2010). Tal es el caso de la Red Mexicana de Investigación Ecológica a Largo Plazo (Red Mex-LTER) creada en de la Red Mexicana de Investigación Ecológica a Largo Plazo (Red Mex-LTER) creada en 2004 como una iniciativa para consolidar la investigación sobre la estructura y el funcionamiento de los ecosistemas terrestres y acuáticos del país. Esta red incluye 11 sitios representativos de distintos ecosistemas de México (Burgos et al., 2007; Maass, Equihua y Jardel, 2008). La investigación que se desarrolla en estos sitios aborda 7 áreas temáticas con un enfoque de investigación socioambiental de largo plazo que ha permitido crear una plataforma de bases de datos para la toma de decisiones (Jardel, Maass y Rivera-Monroy, 2013; Rivera-Monroy et al., 2008). Así mismo, la conformación del Programa Mexicano del Carbono (PMC) en 2005 (Vargas et al., 2012) y de las recientemente creadas redes temáticas del Consejo Nacional de Ciencia y Tecnología (Conacyt), como la Red de Ecosistemas (EcoRed-Conacyt), han estimulado la investigación de corte ecosistémico en nuestro país. Como veremos más adelante, esto se refleja en los artículos publicados en la última década y en el aumento en el número de trabajos que analizan los patrones y procesos en una mayor variedad de tipos de ecosistemas en México, aunque el énfasis sigue siendo en la parte aérea del ecosistema y en el componente de plantas leñosas. El funcionamiento del componente subterráneo ha sido menos estudiado debido a las dificultades metodológicas asociadas al trabajo en el perfil del suelo. En general, dada su complejidad, son aún escasos los estudios integrales de los patrones y procesos del funcionamiento de los ecosistemas tanto en México como a nivel mundial (Escobar et al., 2008).

El presente trabajo responde a la iniciativa de la Sociedad Científica Mexicana de Ecología (SCME), que en 2014 organizó el simposio «La ecología en México: retos y perspectivas». Este esfuerzo de recopilación de información y análisis del estado del arte sobre la estructura y funcionamiento de los ecosistemas terrestres de México es importante porque permite identificar temas que aún no han sido estudiados y estimular la investigación para llenar vacíos de información. Complementa otros esfuerzos de interés similar, como la publicación del libro Capital Natural de México en el 2008 por la Comisión Nacional para el Conocimiento y Uso de la Biodiversidad (Conabio), y otros de carácter más amplio, como la revisión que presenta el 
libro Una mirada al conocimiento de los ecosistemas de México por Balvanera, Arias, Rodríguez-Estrella, Almeida y Schmitter (2016). El trabajo que aquí presentamos está enfocado a la investigación básica sobre 2 grandes temas de la ecología de ecosistemas terrestres: la dinámica hidrológica y los almacenes y flujos de nitrógeno $(\mathrm{N})$ y fósforo $(\mathrm{P})$.

\section{Objetivos}

El objetivo del presente trabajo fue realizar un análisis del estado actual del conocimiento sobre los ecosistemas terrestres de México desde el punto de vista de la dinámica hidrológica y los almacenes y flujos de $\mathrm{N}$ y $\mathrm{P}$, basado en una recopilación de la información disponible en la literatura científica en los últimos 25 años.

Los estudios relacionados con el ciclo del carbono (C) en México, inherentemente vinculado a los ciclos de agua y nutrientes del ecosistema (Hastings, Oechel y Muhlia-Melo, 2005; Vargas et al., 2012) no forman parte de la presente revisión. La información sobre ese tema se puede consultar en las Síntesis Nacionales publicadas por el Programa Mexicano del Carbono (PMC) en su portal de internet (http://pmcarbono.org/pmc/).

Con base en nuestra revisión, presentamos primero una síntesis cuantitativa de la tendencia temporal del número de publicaciones en cada tema analizado (dinámica hidrológica y almacenes y flujos de $\mathrm{N}$ y $\mathrm{P}$ ). Hacemos una revisión de los tipos de ecosistemas y número de sitios estudiados, los objetivos de investigación en cada uno de los 2 temas, de los aportes al conocimiento y de su aplicación para resolver problemas. Continuamos con un análisis de las instituciones académicas mexicanas y del extranjero participantes en las publicaciones, así como de los programas de educación superior en México que ofrecen cursos de Ecología de Ecosistemas y participan en la formación de recursos humanos en este campo. Finalizamos señalando algunos vacíos de conocimiento, retos y perspectivas de investigación sobre estos 2 temas del funcionamiento de los ecosistemas, y una conclusión general.

\section{Métodos}

La presente síntesis está basada en una búsqueda de artículos científicos publicados de 1990 a 2016 en revistas indizadas en la base de datos de la International Science Indexing (ISI) Web of Knowledge (http://apps.isiknowledge. com) y de EBSCO Electronic Journal Service (EJS, http:// ejournals.ebsco.com/Login.asp). Somos conscientes de que existe una gran variedad de fuentes de información (revistas no indizadas, libros, capítulos de libro, reportes, informes, etc.); sin embargo, limitamos la búsqueda a dichas bases de datos por ser de las más completas sobre artículos publicados en revistas indizadas a nivel internacional.

Realizamos la búsqueda usando las palabras clave: ciclo hidrológico, ecohidrología, partición de la precipitación, lluvia, interceptación, escurrimiento, escorrentía, infiltración, evapotranspiración, transpiración, flujos de nutrientes, micorrizas, fijación biológica, nitrógeno, fósforo, amonio, nitrato, ecosistema y México, en español y en inglés. Filtramos la búsqueda para excluir humedales, ecosistemas ribereños y costeros, agroecosistemas y plantaciones. Esto con el fin de centrar nuestro diagnóstico en los ecosistemas naturales terrestres de México, usando como criterio de selección el tipo de vegetación y la descripción de los sitios por los autores de cada estudio, así como el conocimiento propio de los sitios. La búsqueda arrojó un total de 100 artículos indizados (48 sobre la dinámica hidrológica y 52 sobre almacenes y flujos de $\mathrm{N}$ y $\mathrm{P}$ ).

Se capturó la información de cada publicación en una base de datos para organizar los estudios y conocer la contribución de los trabajos por tema, por tipo de ecosistema, objetivos, variables analizadas, lugar, escala espacial y temporal del estudio, año de publicación, nacionalidad de los autores e instituciones participantes. Para conocer los programas docentes y la contribución a la formación de recursos humanos se realizó una búsqueda en las bases de datos disponibles de las instituciones con mayor representación en los estudios de ecología en México. La nomenclatura de los tipos de vegetación en este trabajo está basada en Rzedowski (2006).

\section{Resultados y discusión}

\section{Estado actual del conocimiento sobre la dinámica hidrológica}

Un antecedente importante del estado actual del conocimiento sobre el funcionamiento de los ecosistemas terrestres de México fue el establecimiento de un proyecto a largo plazo sobre la estructura y el funcionamiento de ecosistemas tropicales secos que lleva a cabo la UNAM desde comienzos de 1980 en la Estación de Biología Chamela, ubicada en la costa de Jalisco (Maass et al., 2002; Sarukhán y Maass, 1990). En el marco de este proyecto se dieron los primeros estudios sobre el uso de agua de especies leñosas (Barradas y Fanjul, 1985) y sobre la dinámica hidrológica en microcuencas para analizar el balance de agua en el bosque tropical caducifolio (Cervantes, Maass y Domínguez, 1988; Maass, 1992; Vose y Maass, 1999). Paralelamente, se desarrollaron diferentes estudios de biogeoquímica del bosque (Campo, Jaramillo y Maass, 1998; Campo, Maass, Jaramillo y Martínez-Yrízar, 2000; Campo, Maass, Jaramillo, Martínez-Yrízar y Sarukhán, 2001; Davidson et al., 1993; Davidson et al., 1991; García-Oliva, Casar, Morales y Maass, 1994; García-Oliva, Maass y Galicia, 1995; García-Oliva, Martínez y Maass, 1995; García-Méndez, Maass, Matson y Vitousek, 1991; Maass, 1995; Maass, Jordan y Sarukhán, 1988; Vitousek, Matson, Volkmann, Maass y García, 1989) y se dieron las primeras tesis de licenciatura y posgrado. Algunas síntesis de estos trabajos pioneros se pueden encontrar en Maass et al. (2002), Maass y Burgos (2011) y Jaramillo, Martínez-Yrízar y Sanford (2011).

Desde entonces, la investigación sobre este tema se ha ido robusteciendo y desarrollando en diferentes localidades del país, con estudios enmarcados desde una perspectiva ecohidrológica: un enfoque de las biogeociencias que ha permitido elucidar cómo los procesos hidrológicos tienen una influencia en la distribución, estructura y funcionamiento de los ecosistemas y cómo las respuestas de los procesos bióticos a su vez, a través de 


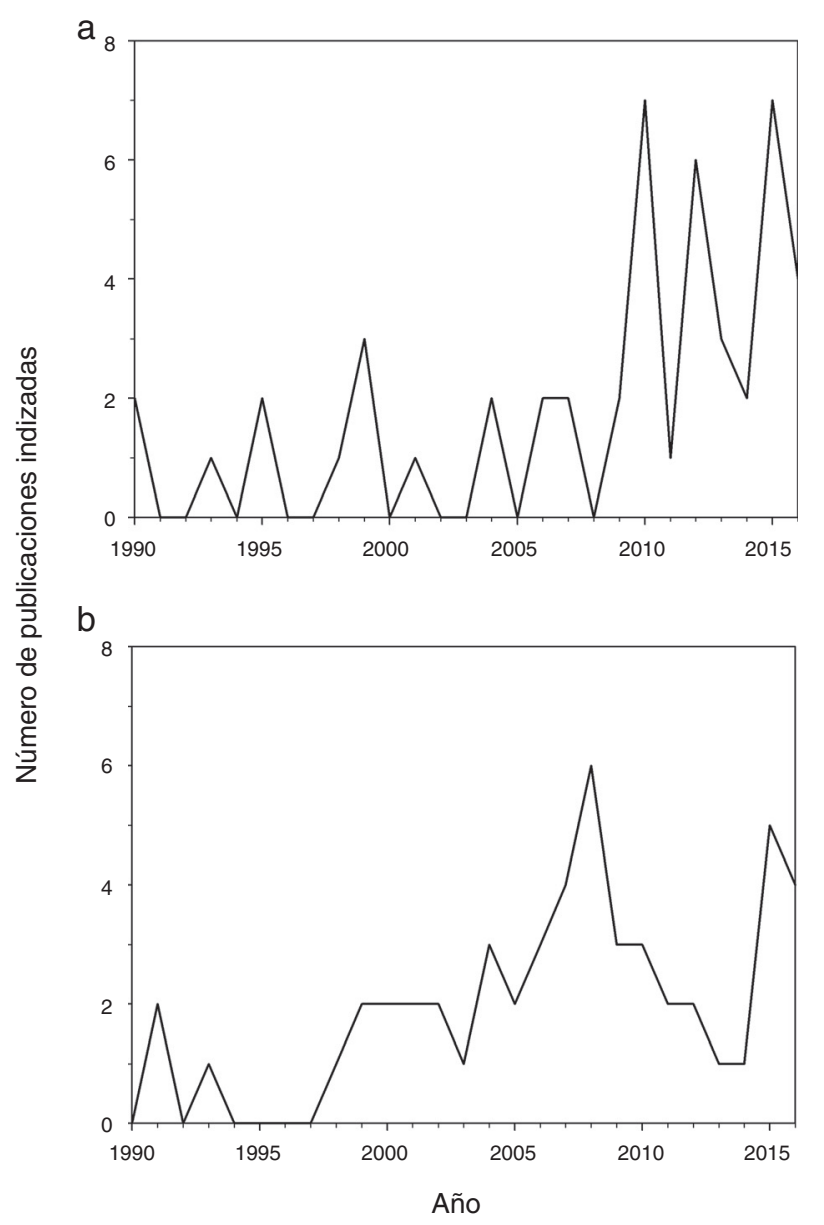

Figura 1. Número de artículos publicados en revistas indizadas entre $1990 \mathrm{y}$ 2016 sobre el funcionamiento de ecosistemas terrestres en México. a) dinámica hidrológica; b) almacenes y flujos de nitrógeno y fósforo.

procesos de retroalimentación, impactan el ciclo hidrológico. Sin embargo, no fue sino hasta el año 2010 que aumentó el ritmo de publicación (63\% del total de los trabajos publicados entre 2000 y 2016), con una tasa muy variable de un año a otro y un máximo de 7 artículos publicados en los últimos 5 años, entre 2010 y 2015 (fig. 1a). Este aumento en el número de trabajos publicados se relaciona, principalmente, con la aplicación de nuevas técnicas, como el uso de torres para el monitoreo continuo de los flujos de vapor de agua y de $\mathrm{CO}_{2}$ (empleando la técnica conocida como «Eddy Covariance») y el uso de isótopos estables para analizar el movimiento del agua de lluvia en el ecosistema y diferenciar el uso de distintas fuentes de agua (precipitación, niebla, agua en el suelo, arroyos y manto freático) por las plantas (Goldsmith et al., 2012; Tarin et al., 2014; Vargas et al., 2013). Esta aproximación ha permitido pasar de una investigación básicamente descriptiva a una orientada a identificar los vínculos y mecanismos involucrados en la dinámica hidrológica y los flujos de elementos en el ecosistema.

Del total de las 48 publicaciones indizadas sobre este tema para el periodo 1990-2016, los matorrales xerófilos son los ecosistemas que han recibido mayor atención de los estudios analizados, representando el $42 \%$ de las publicaciones (fig. 2a). Le siguen los bosques templados, en especial los bosques de

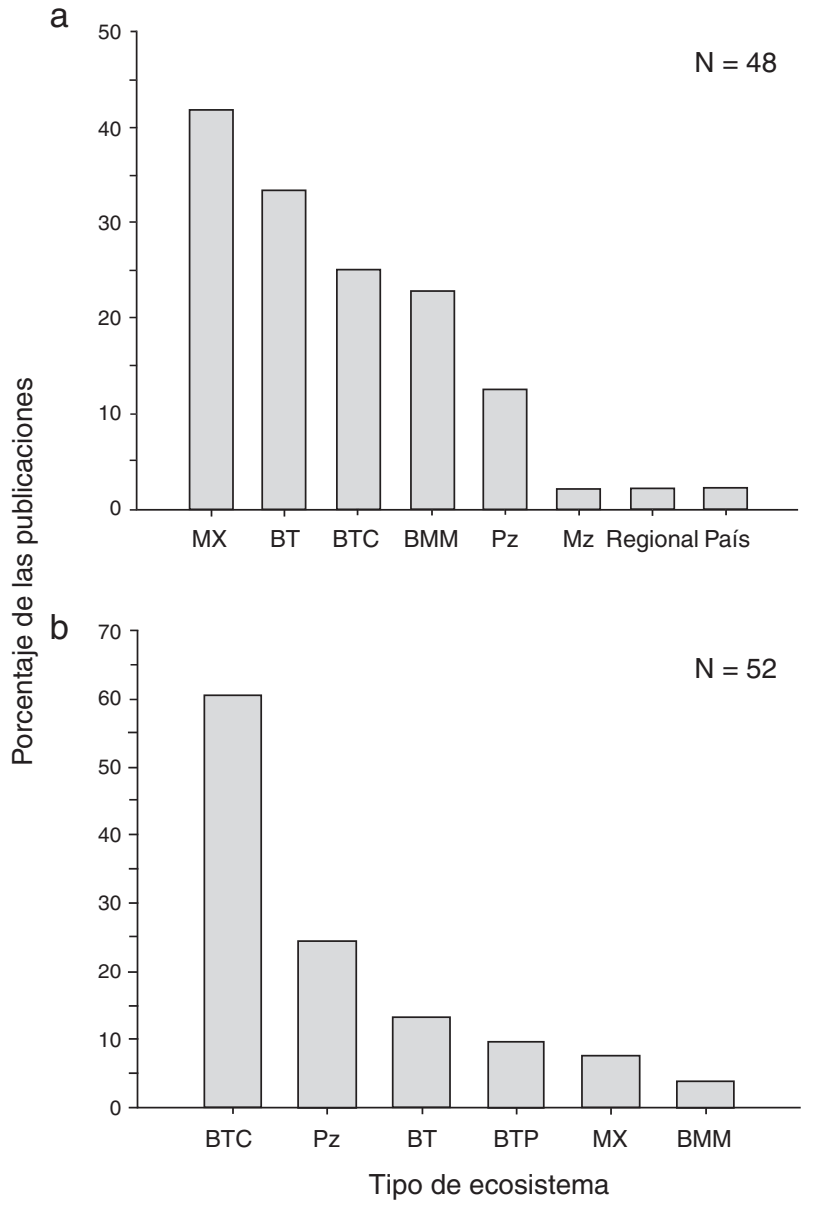

Figura 2. Porcentaje de estudios por tipo de ecosistema del total de las publicaciones indizadas producidas de 1990 a 2016 sobre el funcionamiento de ecosistemas terrestres en México. a) dinámica hidrológica; b) almacenes y flujos de nitrógeno y fósforo.BMM: bosques mesófilos de montaña; BT: bosques templados; BTC: bosques tropicales caducifolios; BTP: bosques tropicales perennifolios; MX: matorrales xerófilos; Mz: mezquitales; Pz: pastizales.

pino, de encino y de pino-encino, presentes en el $33 \%$ de los estudios, y los bosques tropicales caducifolios en el $25 \%$ del total de las publicaciones. Los bosques mesófilos de montaña, en el $23 \%$ de los trabajos, son el ecosistema para los que mejor se conoce el comportamiento de los distintos componentes del flujo de agua, así como la influencia de factores topográficos y de distintos tipos de cobertura (bosques de coníferas vs. bosques de latifoliadas) en la entrada de agua al suelo, la interceptación de niebla, entre otros aspectos clave de la dinámica hidrológica a escala de cuenca (Berry, Gotsch, Holwerda, Muñoz-Villers y Asbjornsen, 2016; Holwerda, Bruijnzeel, Muñoz-Villers, Equihua y Asbjornsen, 2010; Muñoz-Villers, Geissert, Holwerda y McDonnell, 2016). Los pastizales han sido analizados en el 13\% de las publicaciones, en unas cuantas localidades del país. Hasta ahora, un solo estudio en un mezquital cuantificó el uso diferencial de agua por las plantas para explicar los mecanismos de la invasión de Prosopis velutina (mezquite) y las consecuencias del cambio de cobertura en el balance hídrico en áreas donde históricamente predominaban los pastizales semiáridos en el norte de México (Brunel, 2009). 
La mayor atención de los estudios hacia las zonas áridas y semiáridas del país (matorrales y bosques tropicales caducifolios; $67 \%$ de las publicaciones) se debe a que la interacción atmósfera-vegetación-suelo es particularmente intensa en ecosistemas limitados por agua. En estas zonas, la disponibilidad de agua es crítica no únicamente por su escasez, sino también porque es altamente variable e impredecible en tiempo y espacio (Maass et al., 2002; Porporato y Rodríguez-Iturbide, 2002). Para estos ecosistemas ya se tiene un mejor conocimiento de la contribución relativa de los componentes de la evapotranspiración (evaporación del suelo y transpiración) a la variación diaria y estacional de la misma (Robles-Morua, Vivoni y Mayer, 2012; Tarin et al., 2014), así como de la influencia de la estacionalidad contrastante de la precipitación (lluvias de invierno vs. lluvias de verano) en la dinámica de la evapotranspiración (Villarreal et al., 2016).

Respecto a los objetivos de investigación, el $35 \%$ de los estudios han analizado la influencia de la estructura de la vegetación en la «partición de la precipitación», es decir, cómo se mueve y distribuye el agua en el ecosistema, o han analizado la relación entre rasgos funcionales de las plantas (forma, tamaño, fenología, características foliares, etc.) y el uso de agua, con el fin de dilucidar los mecanismos que determinan el balance de agua en el ecosistema (tabla 1). En particular, se ha explorado cómo dichos atributos afectan la cantidad de agua de lluvia que es interceptada, que escurre por los tallos (llamada «escurrimiento por los troncos») y que atraviesa el follaje («escurrimiento por el follaje»), lo que a su vez determina la magnitud de la infiltración y la escorrentía superficial (Cantú-Silva y González-Rodríguez, 2001; Gómez-Tagle, Gómez-Tagle, Ávila y Bruijnzeel, 2015; Kellman y Roulet, 1990; Ponette-González, Weathers y Curran, 2010). Se ha encontrado que los valores de estos flujos varían considerablemente entre tipos de cobertura del suelo e intensidades de lluvia. Por ejemplo, Holwerda et al. (2010) encontraron que la fracción de lluvia anual que se pierde por interceptación (y que no atraviesa el dosel) fue del 17\% en el bosque mesófilo de montaña maduro y solo del $8 \%$ en el bosque mesófilo secundario. Esta diferencia se debió a la mayor capacidad de almacenamiento de agua en el dosel y a la mayor área foliar específica en el bosque maduro. Pérez-Suárez, Arredondo-Moreno, Huber-Sannwald y Serna-Pérez (2014) encontraron que el agua

Tabla 1

Temas de la investigación sobre la dinámica hidrológica en los ecosistemas terrestres de México en artículos indizados para el periodo 1990-2016.

\begin{tabular}{llc}
\hline Tema & $\mathrm{n}$ & $\%$ \\
\hline $\begin{array}{l}\text { Interacción entre partición lluvia, atributos vegetación y } \\
\quad \text { rasgos funcionales }\end{array}$ & 17 & 35.4 \\
$\begin{array}{l}\text { Interacción procesos hidrológicos y actividad vegetación } \\
\text { Disturbios (recarga acuíferos, incidencia fuegos, invasión, }\end{array}$ & 8 & 16.7 \\
$\quad$ cambio de uso del suelo) & 16.7 \\
$\quad \begin{array}{l}\text { Interacción procesos hidrológicos y procesos del } \\
\quad \text { ecosistema }\end{array}$ & 7 & 14.6 \\
$\begin{array}{l}\text { Metodológicos (aplicabilidad de modelos, estrategias de } \\
\quad \text { muestreo) }\end{array}$ & 5 & 10.4 \\
$\begin{array}{l}\text { Experimentales (manipulación de lluvia) y aplicados } \\
\text { Total }\end{array}$ & 3 & 6.3 \\
\hline
\end{tabular}

interceptada, los escurrimientos por el tronco y por el follaje y la escorrentía variaron entre tipos de bosques (monoespecíficos y mixtos de pino-encino) y la intensidad de lluvia en una microcuenca en la mesa central de San Luis Potosí. Identificaron que la distribución de la lluvia en estos bosques es una función de los rasgos morfo-funcionales de cada especie (dimensiones de los árboles), de la estructura del bosque (densidad de árboles, índice de área foliar) y de la interacción entre las especies. Otros estudios comparativos de la influencia de las especies en la dinámica hidrológica del ecosistema incluyen los trabajos de Carlyle-Moses, Flores Laureano y Price (2004), Návar (1993, 2011) y Návar y Bryan (1990) en bosques y matorrales del noroeste de México. En el bosque mesófilo de montaña, el estudio de Alvarado-Barrientos et al. (2015) analizó la contribución de las especies dominantes a la pérdida de agua por transpiración diurna y nocturna. Estos estudios están revelando los vínculos existentes entre diferentes procesos ecohidrológicos (entrada, flujos y almacenes de agua) así como identificar el origen de las fuentes de agua que permiten a los ecosistemas mantener sus funciones a través del tiempo (Goldsmith et al., 2012).

El 15\% de los estudios han investigado los atributos hidrológicos y su influencia en procesos del ecosistema a distintas escalas temporales y espaciales (tabla 1). Por ejemplo, en ecosistemas áridos se ha investigado el efecto de la variación temporal (estacional e interanual) de la precipitación en el intercambio neto de $\mathrm{CO}_{2}$ y de vapor de agua (Pérez-Ruíz et al., 2010; Verduzco et al., 2015), en bosques tropicales secos, el papel de pulsos grandes de lluvia ( $\geq 10 \mathrm{~mm}$ ) en las tasas netas de descomposición (Anaya, Jaramillo, Martínez-Yrízar y García-Oliva, 2012) y la relación entre la variación estacional durante un año de la intercepción potencial de la radiación solar y el contenido de agua del suelo a nivel de cuenca (Galicia, López-Blanco, Zarco-Arista, Filips y García-Oliva, 1999).

El 17\% de los trabajos examinan las interacciones entre los procesos hidrológicos y la actividad de la vegetación, analizada a través de datos derivados de percepción remota (tabla 1). Esta aproximación ha probado ser una herramienta útil para escalar los procesos del ecosistema a nivel de cuenca, regional y de país (Gondwe et al., 2010; Mora e Iverson, 1998; Tang, Vivoni, Muñoz-Arriola y Lettenmaier, 2012; Vivoni, 2012), así como validar/construir modelos predictivos del funcionamiento de ecosistemas basados en procesos hidrológicos (Ballinas, Esperón-Rodríguez y Barradas, 2015; Carlyle-Moses y Price, 2007; Méndez-Barroso et al., 2014). Algunos de estos estudios han examinado la influencia del Monzón de Norteamérica, fenómeno climatológico de gran escala que aporta del 40 al $80 \%$ de la precipitación anual en solo 3 meses durante el verano cada año (Robles-Morua et al., 2012), para explicar las variaciones en la dinámica de la vegetación, la evolución de la evapotranspiración y la partición de la precipitación en ecosistemas áridos y de alta montaña (Méndez-Barroso y Vivoni, 2010; Méndez-Barroso, Vivoni, Watts y Rodríguez, 2009; Tang et al., 2012).

Se ha avanzado en la validación de modelos analíticos de atributos hidrológicos a distintos niveles espaciales y su aplicabilidad a diferentes tipos de cobertura (Ballinas et al., 2015; Návar, 2013; Návar, Carlyle-Moses y Martínez, 1999; Návar, Charles y Jurado, 1999), así como en la evaluación de diseños 
de muestreo (Carlyle-Moses et al., 2004) y estrategias metodológicas para el análisis preciso de la dinámica hidrológica en el ecosistema (Gebremichael y Barros, 2006; Vivoni et al., 2010; $10 \%$ de los trabajos, tabla 1). Este avance ha estado acompañado del uso de torres de flujos turbulentos, de datos provenientes de percepción remota, o de una combinación de ambos; técnicas presentes en el $30 \%$ de las publicaciones.

Las alteraciones de los componentes del flujo de agua asociadas al cambio de uso de suelo, la invasión de especies y la incidencia de disturbios como incendios y plagas son aspectos que han sido abordados en el $17 \%$ de los estudios sobre la dinámica hidrológica en los ecosistemas terrestres de México (tabla 1). Un ejemplo de esto son los trabajos en el bosque mesófilo de montaña, que con la combinación de mediciones ecofisiológicas, micrometeorológicas, hidrológicas y el uso de isótopos estables han podido identificar el impacto hidrológico y las relaciones causales por la conversión del bosque a otros tipos de cobertura (Muñoz-Villers y McDonnell, 2013; MuñozVillers et al., 2015) o el tiempo necesario para la recuperación de la funcionalidad hidrológica del bosque (Muñoz-Villers et al., 2012, 2015).

Los estudios con un enfoque experimental son escasos. Arredondo et al. (2016), a través de un experimento de manipulación de lluvia, mostraron que el pastizal semiárido tropical es tolerante a la sequía pero sensible al legado de la precipitación previa de invierno en la productividad del siguiente verano. Este conocimiento es relevante en el contexto de la vulnerabilidad de la disponibilidad de agua ante el cambio climático y el cambio de uso de suelo (tan intenso en nuestro país).

En los últimos 10 años, la aportación de los estudios en México sobre la dinámica hidrológica ha aumentado, tanto en el número de ecosistemas distintos como en la variedad de localidades de estudio. La investigación se ha realizado en 10 estados de la República Mexicana, siendo Veracruz y Sonora los estados con el mayor número de publicaciones ( $25 \%$ cada uno), con estudios en los bosques mesófilos de montaña de la región central de Veracruz y en Sonora, en los ecosistemas áridos ubicados en las cuencas del río Sonora y del río San Miguel (MéndezBarroso y Vivoni, 2010; Tarin et al., 2014; Vivoni et al., 2010). En Nuevo León (21\% del total de publicaciones) los estudios se han realizado en encinares, bosques de pino, bosques mixtos de pino-encino y en el matorral tamaulipeco en la planicie costera del Golfo de México (Cantú-Silva y González-Rodríguez, 2001; Carlyle-Moses et al., 2004; Návar, 2011; Návar et al., 1999b). El estado de Jalisco (9\% del total de publicaciones) sobresale por los estudios en la región de Chamela, donde predomina el bosque tropical caducifolio (Galicia et al., 1999; García-Oliva et al., 1995a; García-Oliva et al., 1995b). En cada uno de los otros 6 estados se han publicado únicamente uno o dos trabajos sobre este tema (fig. 3a).

\section{Estado actual del conocimiento sobre la dinámica del nitrógeno y fósforo}

Los estudios sobre la dinámica de nutrientes en los ecosistemas terrestres de México han tenido principalmente un enfoque de investigación básica, dirigidos a describir los almacenes y

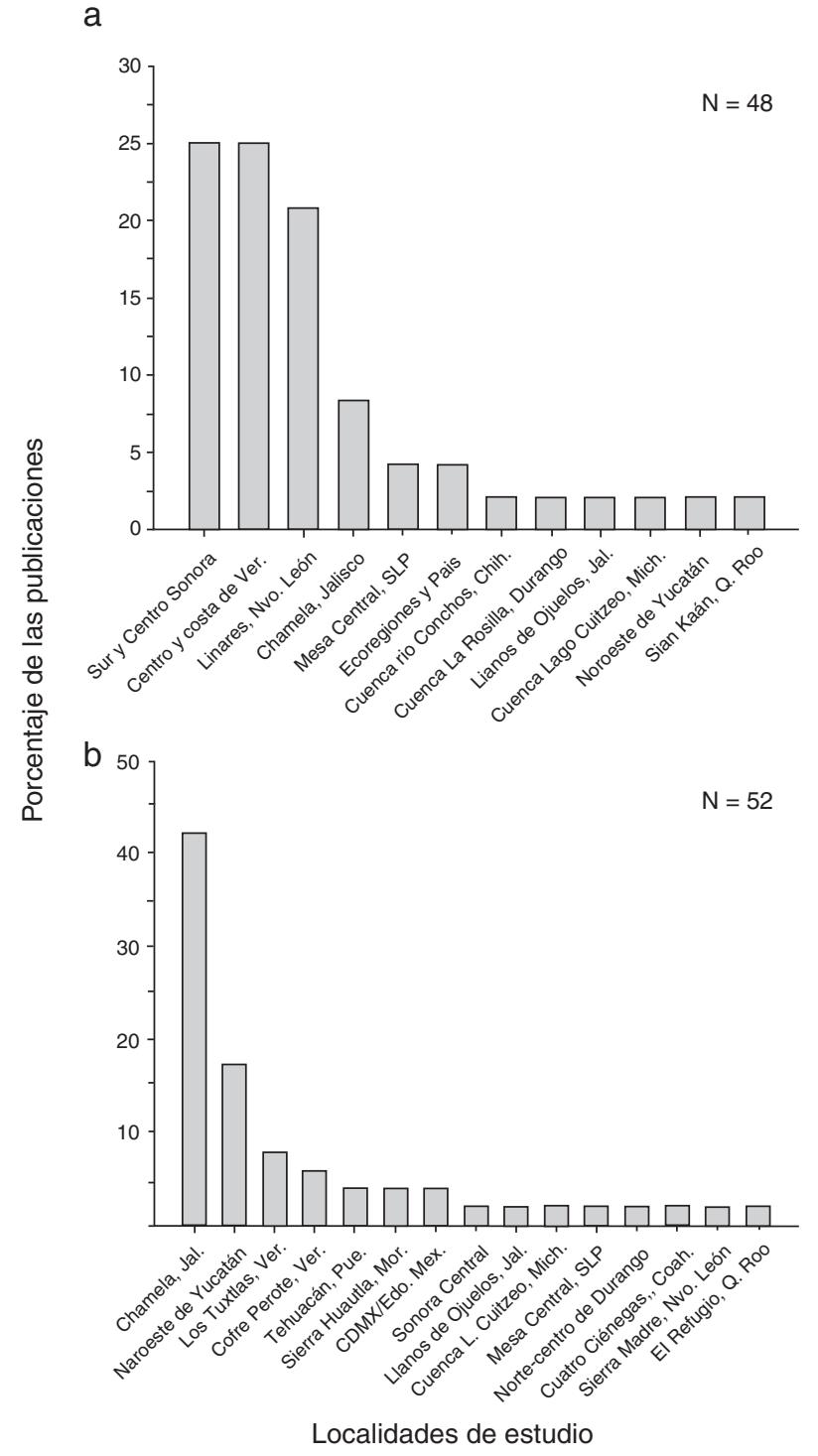

Figura 3. Porcentaje de estudios por localidad del total de las publicaciones indizadas producidas de 1990 a 2016 sobre el funcionamiento de ecosistemas terrestres en México. a) dinámica hidrológica; b) almacenes y flujos de nitrógeno y fósforo.

flujos de C, N y P y la influencia de la variabilidad climática en dichos procesos. De acuerdo con los criterios de la búsqueda para esta revisión, los estudios comenzaron en 1991 con un trabajo en el bosque tropical caducifolio de Chamela sobre las emisiones de óxido nítrico del suelo (García-Méndez et al., 1991). Al igual que los estudios sobre la dinámica hidrológica, el número de publicaciones por año ha sido muy variable, con la mayor producción en los últimos 10 años (65\% del total de las 52 publicaciones de 1990 a 2016) y un máximo de 6 artículos hasta el 2008 (fig. 1b). El ciclo del $\mathrm{N}$ ha sido el más investigado por ser uno de los nutrientes más importantes y limitante de la productividad primaria en muchos ecosistemas terrestres (LeBauer y Treseder, 2008). Así, mientras que el $86 \%$ de los trabajos abordaron aspectos del ciclo del N, solo el $50 \%$ abordó aspectos del ciclo del P. 
Por tipo de ecosistema, en los últimos 25 años los estudios sobre la dinámica del $\mathrm{N}$ y $\mathrm{P}$ se han llevado a cabo en bosques tropicales, bosques templados, pastizales y matorrales xerófilos en muy pocas localidades del país. El bosque tropical caducifolio aparece en el $60 \%$ de las 52 publicaciones, seguido de los pastizales, con el $25 \%$ (fig. 2b). El bosque templado está representado en el $13 \%$ de los estudios, seguido por el bosque tropical perennifolio y el matorral xerófilo, con poco menos del $10 \%$ cada uno. La dinámica de $\mathrm{N}$ y $\mathrm{P}$ en bosques mesófilos de montaña ha sido pobremente estudiada (4\% del total de publicaciones), lo cual contrasta con el alto número de trabajos realizados sobre la dinámica hidrológica de este ecosistema en México, como se mostró en el apartado anterior. El mayor número de trabajos en los bosques tropicales caducifolios se debe, al igual que con los estudios de hidrología, a los esfuerzos e intereses institucionales por conocer el funcionamiento de este tipo de ecosistemas, que cubren aproximadamente el $10 \%$ del territorio, albergan una alta biodiversidad y se encuentran bajo una fuerte presión por disturbio antropogénico (Portillo-Quintero y Sánchez-Azofeifa, 2010). Históricamente, estos esfuerzos se han centrado en la región de Chamela, en la costa de Jalisco, con el propósito de entender los efectos de la estacionalidad y de la conversión del bosque a campos agropecuarios (Jaramillo et al., 2011; Maass et al., 2002). Una aportación reciente de relevancia global al conocimiento de la dinámica de nutrientes en los bosques tropicales de las tierras bajas y de montaña es la publicación de un metaanálisis que exploró las consecuencias del enriquecimiento antropogénico de $\mathrm{N}$ atmosférico en los ciclos del $\mathrm{C}$ y el $\mathrm{N}$. Este análisis cubrió una revisión de 64 estudios repartidos en 39 localidades en 14 países, con una sola localidad en la península de Yucatán representando a México (Bejarano-Castillo, Campo y Roa-Fuentes, 2015).

Por tema de investigación, las publicaciones sobre $\mathrm{N}$ y $\mathrm{P}$ incluyen estudios relacionados con el análisis cuantitativo de los almacenes y flujos de elementos en ecosistemas conservados, la influencia del dosel y de las especies dominantes en estos flujos, así como de las interacciones entre estos nutrientes y la actividad microbiana del suelo (tabla 2). Otros temas relevantes incluyen el efecto de disturbios (cambio de uso del suelo, efecto de borde) y el análisis de los cambios a través de la sucesión secundaria. El compartimento más estudiado es el del suelo (Campos, 2010; Hughes, Kauffman y Jaramillo, 1999, 2000; Jaramillo, Kauffman, Rentería-Rodríguez, Cummings y Ellingson, 2003; Medina-Roldán, Arredondo, Huber-Sannwald, Chapa-Vargas y Olalde, 2008; Saynes, Hidalgo, Etchevers y Campo, 2005), seguido por el del mantillo (Anaya, GarcíaOliva y Jaramillo, 2007; Campo, Solís y Valencia, 2007; Tobón, Martínez-Garza y Campo, 2010). En general, datos sobre los flujos de $\mathrm{N}$ y $\mathrm{P}$ por vía de la caída hojarasca, la principal ruta del regreso de los nutrientes al suelo, son escasos para la mayoría de los ecosistemas terrestres de México.

El enriquecimiento de nutrientes a través del escurrimiento del agua de lluvia por el follaje ha sido abordado en el $17 \%$ del total de las publicaciones (tabla 2). Este flujo se ha documentado para bosques tropicales caducifolios (Campo et al., 1998, 2001; Runyan et al., 2013), bosques templados (Pérez-Suárez, Fenn, Cetina-Alcalá y Aldrete, 2008) y el bosque tropical perennifolio
Tabla 2

Temas de la investigación sobre los almacenes y ciclos de nitrógeno y fósforo en los ecosistemas terrestres de México en artículos indizados para el periodo 1990-2016.

\begin{tabular}{lll}
\hline Tema & $\mathrm{n}$ & $\%$ \\
\hline $\begin{array}{l}\text { Efecto de disturbios (cambio de uso } \\
\text { del suelo, efecto de borde) }\end{array}$ & 15 & 28.8 \\
$\begin{array}{l}\text { Influencia del dosel, flujos en } \\
\quad \text { escurrimiento troncal y por el } \\
\text { follaje }\end{array}$ & 9 & 17.3 \\
$\begin{array}{l}\text { Influencia de especies dominantes y } \\
\quad \text { rasgos foliares } \\
\text { Relación con la dinámica microbiana } \\
\quad \text { del suelo }\end{array}$ & 7 & 13.5 \\
$\begin{array}{l}\text { Manejo de ecosistemas (restauración, } \\
\text { fertilización) }\end{array}$ & 7 & 13.5 \\
$\begin{array}{l}\text { Dinámica a través de la sucesión } \\
\text { secundaria }\end{array}$ & 6 & 11.5 \\
$\begin{array}{l}\text { Eficiencia de uso y disponibilidad de } \\
\text { nutrientes }\end{array}$ & 5 & 9.6 \\
Total & 3 & 5.8 \\
\hline
\end{tabular}

(Álvarez-Sánchez, Barajas-Guzmán, Campo y León, 2016). La contribución del enriquecimiento de nutrientes por este flujo varía entre eventos individuales de lluvia, entre compuestos químicos y entre tipos de ecosistema. Por ejemplo, los valores más altos se presentan en los bosques templados, donde los escurrimientos de nitratos a través del follaje pueden alcanzar valores de hasta $3.01 \mathrm{mg} \mathrm{L}^{-} 1$. Al parecer, tales escurrimientos (o flujos) fueron determinados por la forma monopódica de los árboles más que por la cantidad de la precipitación. En los bosques tropicales, tanto estacionales como húmedos, los valores máximos de dichos flujos fueron considerablemente más bajos ( 0.40 y $1.16 \mathrm{mg} \mathrm{L}^{-} 1$, respectivamente).

La influencia de las especies en el flujo de nutrientes, analizada en el $14 \%$ de las publicaciones (tabla 2), incluye los trabajos con especies de pino y abeto en los bosques templados cercanos a la Ciudad de México (Fenn, de Bauer, QuevedoNolasco y Rodríguez-Frausto, 1999; Pérez-Suárez et al., 2008); con las leguminosas Lysiloma microphyllum Benth. (Cárdenas y Campo, 2007) y Caesalpinia eriostachys Benth. (Toledo-Aceves y García-Oliva, 2008) en los bosques tropicales caducifolios de Huautla y Chamela, respectivamente; con Quercus sp. en los bosques de Michoacán (Chávez-Bergara et al., 2016), con Prosopis juliflora (Sw.) DC., Acacia farnesiana (L.) Willd. y Mimosa biuncifera Benth. de la región semiárida de Durango (Herrera-Arreola, Herrera, Reyes-Reyes y Dendooven, 2007) y con Astrocaryum mexicanum Liebm., una palma dominante del sotobosque en el bosque tropical perennifolio de Los Tuxtlas, Veracruz (Álvarez-Sánchez et al., 2016). En este último caso, la influencia de la palma en los ciclos del $\mathrm{N}$ y el $\mathrm{P}$ es notable, ya que sus copas actúan como una trampa natural de la hojarasca que cae de los estratos superiores del bosque formando una capa de mantillo que enriquece con nutrientes el agua de lluvia que escurre por los troncos. Este mecanismo es una ruta significativa en las entradas de $\mathrm{N}$ y $\mathrm{P}$ al suelo en este bosque lluvioso.

Una contribución importante a los estudios sobre la dinámica de nutrientes en los bosques tropicales estacionalmente 
secos ha sido la medición, previo a la abscisión foliar, de la reabsorción (el regreso de nutrientes de las hojas a la planta durante la senescencia foliar) y la proficiencia (concentración de nutrientes en las hojas completamente senescentes) de $\mathrm{N}$ y $\mathrm{P}$ en especies dominantes o por grupos funcionales (Cárdenas y Campo, 2007; Rentería, Jaramillo, Martínez-Yrízar y PérezJiménez, 2005; Rentería y Jaramillo, 2011). Este enfoque ha permitido identificar el control de la disponibilidad de agua en la dinámica de los nutrientes, estudiar la eficiencia de uso de nutrientes y explicar el funcionamiento del ecosistema en términos de los mecanismos de conservación de $\mathrm{N}$ y $\mathrm{P}$ en ambientes estacionalmente secos (Rentería y Jaramillo, 2011). A pesar de la importancia de la reabsorción en la eficiencia de uso y conservación de nutrientes, este es un tema que ha sido muy poco abordado en los estudios sobre la dinámica de nutrientes en los ecosistemas terrestres de México $(6 \%$ del total de las publicaciones).

Los estudios en relación con la actividad microbiana en el suelo (14\% de las publicaciones; tabla 2) se han realizado principalmente en ecosistemas limitados por agua, como bosques secos y matorrales. Se ha estudiado la dinámica de $\mathrm{N}$ en las llamadas islas de fertilidad (Perroni-Ventura, Montaña y García-Oliva, 2006) y en las costras biológicas del suelo (Sandoval-Pérez et al., 2016), mostrando que la biomasa y la actividad microbiana en las islas y las biocostras mejora localmente la dinámica de $\mathrm{C}$ y $\mathrm{N}$ en estos ambientes. Se ha analizado el efecto diferencial de los macro- y micro-agregados del suelo en la dinámica de $\mathrm{C}$ y $\mathrm{N}$, y en la estructura de la comunidad microbiana del suelo en el bosque tropical caducifolio (Noguez et al., 2008), así como el efecto de la composición de la hojarasca en la actividad microbiana y comunidad de hongos en un bosque deciduo de Quercus (Chávez-Bergara et al., 2016). También se ha investigado la influencia de distintas especies de árboles remanentes en pastizales inducidos sobre el $\mathrm{N}$ y $\mathrm{C}$ microbiano del suelo (Galicia y García-Oliva, 2004, 2008). El potencial de transformación de $\mathrm{C}, \mathrm{N}$ y $\mathrm{P}$ por la comunidad microbiana del suelo en dos sistemas vegetación-suelo contrastantes (matorral y pastizal) fue analizado por Tapia-Torres, López-Lozano, Souza y García-Oliva (2015), mostrando que el suelo del pastizal promueve un sistema cerrado que conserva el $\mathrm{N}$, mientras que en el matorral es un sistema es abierto que libera N. Para el bosque tropical caducifolio, Montaño, García-Oliva y Jaramillo (2007) analizaron el efecto de las variaciones estacionales del C orgánico disuelto en la dinámica del $\mathrm{N}$ y la actividad microbiana del suelo. Recientemente se han introducido nuevos enfoques (filogenéticos y moleculares) para analizar, por ejemplo, la relación del $\mathrm{N}$ almacenado en la hojarasca producida por varias especies arbóreas con sus afinidades filogenéticas (Bhaskar, Porder, Balvanera y Edwards, 2016).

Estudios con un enfoque experimental (12\% de las publicaciones; tabla 2) han sido diseñados para analizar el efecto de la fertilización con $\mathrm{N}$ y $\mathrm{P}$ en los almacenes y ciclos de dichos elementos a través de la sucesión secundaria o a través de un gradiente de precipitación. Tal es el caso de los trabajos de Campo, Solís y Gallardo (2012), Ceccon, Sánchez y Campo (2002), Gamboa et al. (2010) y Bejarano, Etchevers, Ruíz-Suárez y Campo (2014) llevados a cabo en bosques tropicales secos.
A través de un experimento de fertilización en el laboratorio, Bejarano et al. (2014) analizaron los efectos sobre los flujos de $\mathrm{NO}_{2}$, la mineralización de $\mathrm{N}$ y la concentración de $\mathrm{N}$ en la biomasa microbiana al añadir $\mathrm{N}$ al suelo proveniente de localidades de bosque tropical seco de Yucatán con diferentes niveles de precipitación. Los resultados mostraron que tales efectos dependen de las condiciones de limitación de agua que operan en cada localidad.

El efecto del cambio de uso del suelo sobre los almacenes y la dinámica de los nutrientes es un tema de estudio que ha recibido una notable atención (29\% de las publicaciones; tabla 2). Ejemplos de estos estudios son los trabajos de Campos (2010), Campos, Etchevers, Oleschko e Hidalgo (2012) y Campos, Oleschko, Etchevers e Hidalgo (2007) en los bosques de niebla del Cofre de Perote, de Valdespino, Romualdo, Cardenazzi y Campo (2009) en los bosques secos de la Sierra de Huautla, de MoralesRomero, Campo, Godínez-Álvarez y Molina-Freaner (2015) en el matorral xerófilo de Sonora, y de García-Oliva, Gallardo y Montaño (2006), Giardina, Sanford y Døckersmith (2000) y Jaramillo et al. (2003) en los bosques estacionalmente secos de Chamela. En particular, se ha encontrado que el $\mathrm{N}$ orgánico del suelo (García-Oliva et al., 2006), la mineralización del N (Campos et al., 2007; Morales-Romero et al., 2015) y los almacenes de N y P (Hughes et al., 2000; Das, Lawrence, d'Odorico y DeLonge, 2011) se reducen como consecuencia de la conversión a campos agropecuarios. Un solo estudio, que integró varios componentes de la dinámica del ecosistema y variables sociales, ha analizado la resiliencia del bosque tropical seco al cambio de uso de suelo (Gavito et al., 2014). Este estudio mostró la buena capacidad de respuesta y adaptación del socio-ecosistema de bosque tropical seco en Chamela, resaltando la importancia que ha tenido la preservación del capital natural, favorecida por la existencia de un área natural protegida y el bajo impacto humano en esa zona (Gavito et al., 2014).

A la fecha, son muy pocas las localidades (distribuidas en 13 estados del país) donde se ha estudiado la dinámica del $\mathrm{N}$ y $\mathrm{P}$ de los ecosistemas terrestres de México (fig. 3b). La mayoría de los trabajos se han realizado en Jalisco (44\% de las publicaciones), seguido por la península de Yucatán (17\%), especialmente en el bosque tropical caducifolio en ambos estados. Con una menor representación, este tema se ha investigado en el estado de Veracruz, principalmente en el bosque mesófilo de montaña y el bosque tropical perennifolio (13\% del total de las publicaciones; fig. 3b). En cada uno de otros 10 estados de la República Mexicana se han publicado uno o dos trabajos sobre los almacenes y dinámica de $\mathrm{N}$ y $\mathrm{P}$ (fig. 3b) y la mayoría en sitios ubicados en comunidades de zonas áridas y semiáridas.

\section{Aportes en la aplicación del conocimiento}

La presente revisión nos permitió identificar varios trabajos que podrían incidir en la aplicación del conocimiento, pero la mayoría $(85 \%)$ han sido de investigación básica. Estudios de corte más aplicado son trabajos que no cumplieron con los criterios de la búsqueda, pero abordan aspectos como la problemática de uso de agua asociada al turismo en las zonas tropicales 
estacionales (Riensche, Castillo, Flores-Díaz y Maass, 2015), el papel de los ecosistemas en la provisión de servicios ecosistémicos (Balvanera et al., 2016), las relaciones entre variables biofísicas y socioeconómicas para elucidar los vínculos entre la degradación del suelo y el ciclo hidrológico a distintas escalas (Huber-Sannwald, Maestre, Herrick y Reynolds, 2006) y la aplicación del enfoque socioecosistémico en la política pública del país (Challenger, Bocco, Equihua, Lazos-Chavero y Maass, 2015). Estos son algunos ejemplos de investigación aplicada que merece un análisis más completo en una futura revisión sobre este tema.

Uno de los estudios identificados en la presente revisión con el potencial de incidir en la aplicación del conocimiento es el trabajo hidrogeológico de Gondwe et al. (2010) en la región de la península de Yucatán donde se ubica la Reserva de la Biosfera de Sian Ka' an. Con el fin de desarrollar una gestión adecuada del agua subterránea, este estudio empleó la combinación de parámetros geofísicos y de percepción remota en la determinación de la recarga de acuíferos (17\% de la precipitación promedio anual) y su variación espacial a través de la región de estudio. Otro trabajo, en el contexto del manejo de bosques, identificó los vínculos entre los periodos de sequía, la humedad del suelo y la incidencia de plagas (escarabajos descortezadores) en áreas quemadas de bosques templados sujetos a una alta incidencia de incendios (Návar, 2015). Este estudio enfatizó que el entender la convergencia de estos vínculos con anomalías climáticas es crítico para evaluar la vulnerabilidad de los bosques a este tipo de disturbios e implementar mejores prácticas de manejo forestal. A escala de cuenca, Muñoz-Villers et al. (2016) cuantificaron el tiempo de tránsito del agua de lluvia en la cuenca alta del río La Antigua, Veracruz, identificando las variables físicas (fisiografía, topografía y permeabilidad de la interfase suelo-roca) que contribuyen a sostener el flujo del caudal, bajo diferentes tipos de cobertura e intensidades de lluvia. Este estudio muestra el valor que tiene la conservación del bosque mesófilo de montaña para mantener los tiempos de residencia del flujo base de ríos largos. Para la cuenca del río Conchos, Chihuahua, Reyes-Gómez, Viramontes-Pereida, Miranda-Ojeda, SánchezFernández y Viramontes-Olivas (2007) compararon la capacidad de infiltración y escorrentía entre suelos de bosque y de pastizal para determinar las áreas de recarga en la cuenca. Este estudio encontró que la infiltración del bosque (intervalo de variación del 60 al 100\%) disminuye al 40\% debido a la deforestación. En el caso del $\mathrm{N}$ y el $\mathrm{P}$, un estudio examinó los efectos de distintos tratamientos de restauración en la recuperación del estatus de los nutrientes del suelo en una pradera ganadera en un sitio anteriormente cubierto por bosque tropical perennifolio (Tobón et al., 2010).

En las 48 publicaciones sobre la dinámica hidrológica de los ecosistemas terrestres de México es notable la colaboración que se ha dado entre instituciones nacionales y del extranjero, tanto de Latinoamérica como de Europa, Australia, Estados Unidos y Canadá. En total, 18 instituciones mexicanas han realizado publicaciones con la colaboración de 30 instituciones del extranjero. Destacan por sus contribuciones 5 instituciones mexicanas, principalmente la UNAM (33\% de las publicaciones), y 2 del extranjero (la Universidad Estatal de Arizona y la Universidad

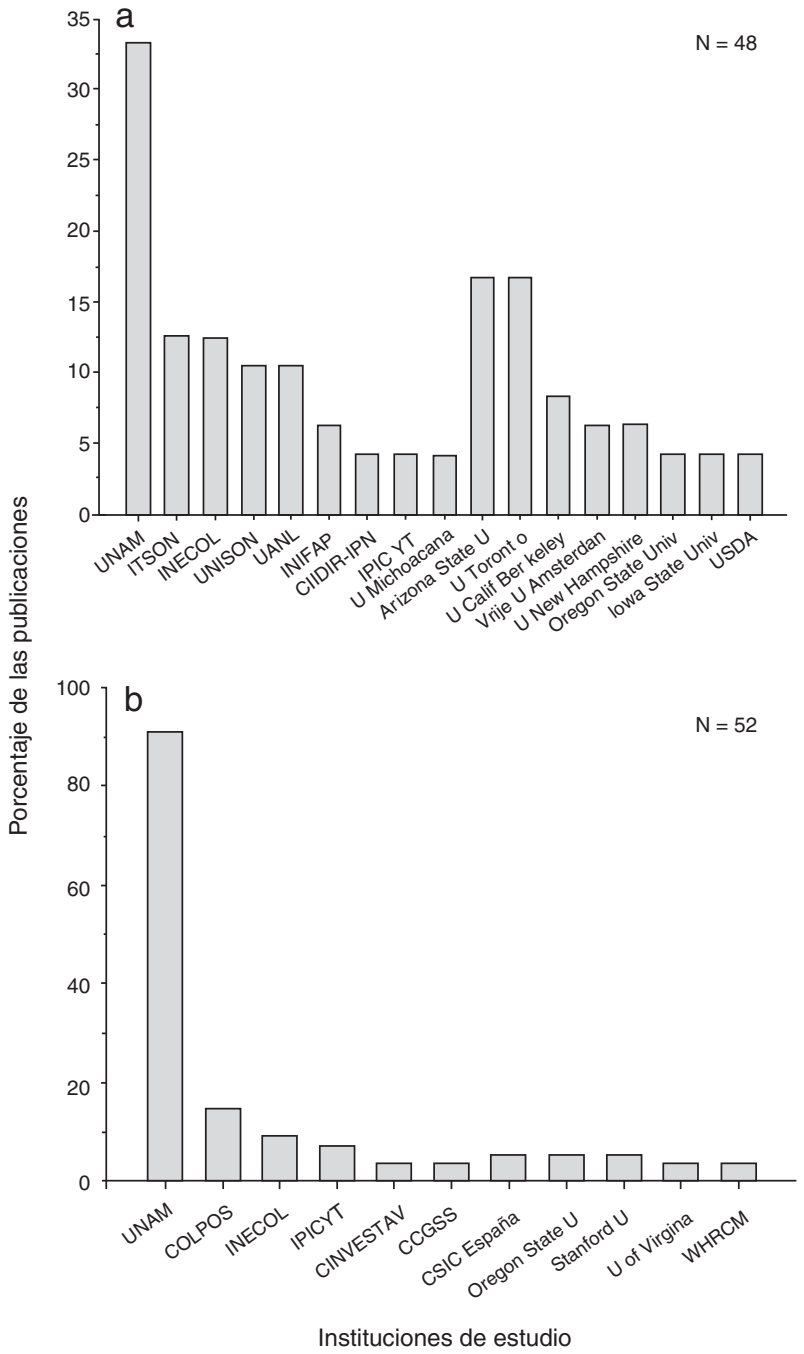

Figura 4. Porcentaje de estudios por institución académica participante del total de las publicaciones indizadas producidas de 1990 a 2016 sobre los ecosistemas terrestres de México. a) dinámica hidrológica; b) almacenes y flujos de nitrógeno y fósforo.CCGSS: Centro del Cambio Global y la Sustentabilidad en el Sureste A.C.; CIIDIR: Centro Interdisciplinario de Investigación para el Desarrollo Regional Integral; CINVESTAV: Centro de Investigación y de Estudios Avanzados del Instituto Politécnico Nacional; CSIC: Consejo Superior de Investigaciones Científicas; INECOL: Centro Instituto de Ecología, A.C.; INIFAP: Instituto Nacional de Investigaciones Forestales, Agrícolas y Pecuarias; IPICYT: Instituto Potosino de Investigación Científica y Tecnológica, A.C.; ITSON: Instituto Tecnológico de Sonora; UANL: Universidad Autónoma de Nuevo León; USDA: U.S. Department of Agriculture; WHRC: Woods Hole Research Center.

de Toronto, con el $17 \%$ de las publicaciones cada una; fig. 4a). Veinte de las 48 publicaciones fueron producto de la colaboración de instituciones mexicanas y del extranjero, 16 fueron producidas solo por instituciones mexicanas y 12 solo por instituciones del extranjero.

Las publicaciones encontradas en esta revisión sobre los almacenes y ciclos de $\mathrm{N}$ y P de los ecosistemas terrestres de México se realizaron con la participación de 25 instituciones: 10 mexicanas y 15 del extranjero. La UNAM está presente en la mayoría de las 52 publicaciones (91\%), seguida por el Colegio de Posgraduados (COLPOS), con un 15\%, y el Instituto de Ecología A.C. (INECOL), con un 9\% (fig. 4b). El resto 
de las 7 instituciones mexicanas están representadas en una o dos publicaciones. Del total de las 52 publicaciones, la mayoría $(71 \%)$ fueron producidas solo por instituciones mexicanas y únicamente 14 publicaciones resultaron de la colaboración internacional, principalmente con el Consejo Superior de Investigaciones Científicas de España, la Universidad Estatal de Oregon y la Universidad de Stanford. Únicamente 2 de los 52 trabajos fueron publicados sin la participación de instituciones mexicanas.

\section{Programas docentes que imparten cursos de Ecología de Ecosistemas en México}

Los cursos de ecología de ecosistemas en México comenzaron a mediados de la década de 1980, cuando inició el Programa de Doctorado en Ecología coordinado por el Centro de Ecología de la Universidad Nacional Autónoma de México (hoy Instituto de Ecología, UNAM) que incluía en su plan de estudios la materia de Ecología de Ecosistemas. Desde 1990, dicho curso se ha impartido de manera ininterrumpida en el Posgrado en Ciencias Biológicas de la UNAM. Hasta donde sabemos, y quizá solo con algunas excepciones (como la Licenciaturas en Ecología de la Universidad Estatal de Sonora [UES] y la de Ingeniero en Recursos Agropecuarios del CUCSUR de la Universidad de Guadalajara), es raro que la materia de Ecología de Ecosistemas, con un contenido temático que abarque marcos conceptuales y todos los componentes del funcionamiento del ecosistema y sus interacciones (hidrología, energética y biogeoquímica), se incluya en los planes de estudios de las licenciaturas afines tales como la de Ciencias de la Tierra en la Facultad de Ciencias de la UNAM y las de Biología en la Universidad Autónoma Metropolitana, en la Universidad de Sinaloa y en la UNAM, entre otras. En estas últimas solo se imparten conocimientos generales de ecología de ecosistemas dentro del programa general de la materia de Ecología, o de cursos independientes por temas, o que combinan la ecología de comunidades y de ecosistemas en una sola materia. Con el surgimiento de carreras más interdisciplinarias y multi-institucionales han ido apareciendo cursos claramente enmarcados en el paradigma ecosistémico. Son un ejemplo de esto los cursos de Hidrología y Energética de Ecosistemas, de Biogeoquímica del Ecosistema y de Manejo Integral de Ecosistemas, que forman parte del plan de estudios de la Licenciatura en Ciencias Ambientales de la Escuela Nacional de Estudios Superiores Unidad Morelia de la UNAM. Un número significativo de tesis de maestría y doctorado, en temas de la dinámica hidrológica y de los almacenes y flujos de nutrientes en los ecosistemas terrestres de México, ha ido aumentado en correspondencia con el mayor número de artículos publicados en estos temas. Por ejemplo, en los catálogos digitales de tesis de posgrado disponibles en los portales de la UNAM, del Instituto Potosino de Investigación Científica y Tecnológica A.C. (IPICYT), del INECOL y del Instituto Tecnológico de Sonora (ITSON) identificamos (basándonos en el título de la tesis) al menos una centena de trabajos que abordaron tópicos relacionados con la dinámica hidrológica y biogeoquímica de los ecosistemas terrestres de México. Estos trabajos de tesis se realizaron bajo la dirección de varios de los investigadores que aparecen como autores en las publicaciones de la presente revisión, la mayoría en los últimos 10 años. Dada la importancia que tiene el tema del agua en los aspectos biológicos, ecológicos y sociales, sobresale en las tesis que abordan temas de la dinámica hidrológica un vínculo con procesos de cambios de uso del suelo y con la provisión y calidad del agua. Sin embargo, aún se requiere un mayor impulso en la formación de recursos humanos en las investigaciones con un enfoque integral al estudio de los patrones y procesos del funcionamiento de los ecosistemas terrestres de México. Tal formación es importante para subsanar el reducido número de expertos que se tienen en el país en este campo de estudio. Como bien lo señalan Vargas et al. (2012), en México esta disciplina se trabaja con una infraestructura y programas de monitoreo de largo plazo aún muy limitados. Esto resulta contradictorio, dada la importancia del enfoque ecosistémico para abordar y resolver los retos ecológicos y ambientales actuales asociados al acelerado cambio en los ecosistemas a nivel nacional y global.

\section{Vacíos del conocimiento, retos y perspectivas de investigación}

Los siguientes son algunos aspectos importantes que se desprenden de la presente revisión de la bibliografía de trabajos de corte ecosistémico en México en los últimos 25 años:

1. Los estudios no solo son escasos, sino que están concentrados en pocas localidades y la mayoría son de corta duración (un año o menos en el $48 \%$ del total de las 100 publicaciones). Dadas las marcadas fluctuaciones temporales de las variables que controlan los procesos del ecosistema (p.ej., la humedad del suelo puede variar de manera relevante a intervalos de horas a décadas), el desarrollo de diseños de largo plazo permitiría determinar con mayor precisión la magnitud de la variabilidad natural y las respuestas/recuperación de los ecosistemas a eventos extremos del clima y disturbios naturales de gran escala (como los eventos El Niño, el Monzón de Norteamérica y la Oscilación Decadal del Pacífico). Tal información es fundamental para entender la vulnerabilidad de los ecosistemas terrestres al cambio climático global.

2. La mayoría de los estudios se han realizado a nivel de un sitio representativo del ecosistema de estudio, mismo que es intensamente monitoreado a nivel de parcela, generalmente en áreas de menos de 0.1 ha. Algunos estudios han abarcado una mayor escala espacial con varios sitios distribuidos en la ladera de un volcán, o a nivel de una cuenca, o en varias subcuencas, pero un número aún muy limitado ha abarcado una escala regional o nacional (4\% del total de publicaciones). Queda aún el reto de que los resultados de estudios a nivel de parcela sean integrados a escalas mayores (p.ej., con modelos predictivos de escalamiento basados en principios teóricos) que permitan predecir/modelar la variabilidad espacial a distintas escalas (local, regional, país) y analizar la universalidad de los patrones observados.

3. El papel que juegan los rasgos funcionales de las plantas en la partición de la precipitación, en la distribución de la 
humedad y de los nutrientes del suelo es un tema que se ha estudiado en muy pocos sitios. Este enfoque constituye un desafío dada la enorme variabilidad que existe en diversidad y estructura dentro y entre tipos de ecosistemas. Sin embargo, es un aspecto que debe atenderse, como línea base de comparaciones con datos empíricos de los mecanismos involucrados en los procesos y en la elaboración teórica posterior.

4. Se requiere un mayor número de estudios para documentar los almacenes de nutrientes y su dinámica asociada a las comunidades de microorganismos del suelo. Este es un tema central considerando que un alto porcentaje del $\mathrm{C}$ de la planta se dirige al mantenimiento de la biomasa de organismos simbiontes, como bacterias y hongos micorrizógenos. La cuantificación de la fijación simbiótica y no simbiótica de $\mathrm{N}$ en los ecosistemas es un tema en progreso (González-Ruíz, Jaramillo, Peña-Cabriales y Flores, 2008); sin embargo, dada la importancia de estos procesos en la entrada de $\mathrm{N}$ al ecosistema, la dinámica de la nodulación bacteriana y los factores que inhiben la actividad de los nódulos son aspectos que requieren una mayor atención de estudio.

5. Sabemos poco sobre la respuesta a los cambios inducidos por disturbios naturales y antropogénicos, en la dinámica hidrológica y los almacenes y flujos de $\mathrm{N}$ y $\mathrm{P}$, en la mayoría de los ecosistemas terrestres de México. Si bien existen algunos trabajos sobre las alteraciones a los ciclos de nutrientes por erosión del suelo asociado a las actividades humanas y sobre los cambios de los flujos y almacenes de $\mathrm{N}$ y $\mathrm{P}$ a través de la sucesión secundaria, son aún contados los estudios con estos enfoques que pueden permitir identificar las tasas de recuperación de los ecosistemas ante disturbios múltiples. Falta, en general, una mayor incorporación a los estudios ecosistémicos de los componentes económico y social como conductores de cambio de los ciclos de agua y nutrientes.

6. Los trabajos relacionados con el cambio climático global (CCG) y con los eventos climáticos extremos en general han sido escasos en México. Dado el contexto climático de los estudios de ecohidrología, en muchos de ellos se plantea la necesidad de conocer los componentes del ciclo hidrológico en el balance de agua como información básica para predecir los impactos del CCG. Sin embargo, en la presente revisión no detectamos trabajos explícitamente diseñados para evaluar los impactos del CCG, excepto por un trabajo que analiza la respuesta de variables fisiológicas asociadas con el uso de agua de las plantas ante distintas condiciones ambientales, como una aproximación para evaluar la vulnerabilidad ambiental regional (Esperón-Rodríguez y Barradas, 2015). Por lo tanto, el desarrollo de experimentos y estudios de modelación en un mayor número de ecosistemas permitiría explorar, en el contexto de escenarios futuros de $\mathrm{CCG}$, las consecuencias de las alteraciones presentes a los ciclos de agua y nutrientes.

7. Enfrentamos aún el reto de la estandarización de metodologías. Un primer esfuerzo al respecto se ha impulsado a través del trabajo en redes nacionales (como la Red MexLTER y el Programa Mexicano del Carbono), con el que se busca que todos los sitios donde se desarrolla investigación de corte ecosistémico, sean equipados siguiendo un mismo protocolo de medición de los procesos y que los datos sean comparables. Sin embargo, estas iniciativas aún están en proceso de consolidarse y lidian con importantes limitaciones de financiamiento.

8. Finalmente, aún se tiene que recorrer mucho camino para que el conocimiento generado sobre el funcionamiento de los ecosistemas terrestres sea utilizado en México en la toma de decisiones sobre la gestión del agua, la protección de la biodiversidad y las funciones y servicios ecosistémicos (p.ej., captación de agua, regulación de inundaciones, control de erosión, protección del suelo) y el manejo de ecosistemas. Habrá que redoblar esfuerzos en la formación de recursos humanos y en la generación de conocimiento e información funcional de corte ecosistémico, como una estrategia de investigación ante la urgente necesidad de atender en el país la severa crisis ambiental que tenemos enfrente.

\section{Conclusiones}

Existen pocos trabajos, artículos en revistas indizadas en los últimos 25 años, sobre la dinámica hidrológica, los ciclos y los almacenes de $\mathrm{N}$ y $\mathrm{P}$, y aún menos los que estudian las interacciones agua-nutrientes en los ecosistemas terrestres de México, tanto desde una perspectiva empírica, experimental, teórica y de modelación. Los estudios no solo son escasos, sino que están concentrados en unas cuantas localidades de pastizales, matorrales xerófilos, bosques templados, bosques de niebla y bosques tropicales secos y lluviosos. La mayoría de los trabajos de monitoreo en campo son de corto plazo $(<10$ años $)$ y a escala espacial de parcela o de microcuenca $\left(<0.3 \mathrm{~km}^{2}\right)$, pero ya se tiene un mejor entendimiento de la magnitud y variabilidad intra e interanual de la dinámica hidrológica, especialmente en los ecosistemas limitados por agua y en los bosques de niebla. La incorporación de metodologías más sofisticadas y el trabajo en redes nacionales han permitido fortalecer la investigación ecosistémica en un mayor número de instituciones académicas en el país y con mayor participación internacional. Un entendimiento de cómo operan estos procesos es aún necesario para diseñar e implementar programas de conservación y manejo integral de los ecosistemas terrestres de México en el largo plazo.

\section{Agradecimientos}

A Enriquena Bustamente, Raúl Ahedo y Salvador Araiza por su apoyo técnico en diferentes etapas del desarrollo del trabajo. A Víctor J. Jaramillo, Alberto Búrquez, Miguel Martínez Ramos y dos revisores anónimos por sus valiosos comentarios que enriquecieron y permitieron mejorar este manuscrito. Este trabajo se realizó durante la estancia sabática de AMY en la Universidad de Arizona (2016-2017) y agradece el apoyo del Programa de Apoyos para la Superación de Personal Académico de la DGAPA, UNAM, y a David Breshears su disposición y apoyo. 


\section{Referencias}

Alvarado-Barrientos, M. S., Holwerda, F., Geissert, D. R., Muñoz-Villers, L. E., Gotsch, S. G., Asbjornsen, H., et al. (2015). Nighttime transpiration in a seasonally dry tropical montane cloud forest environment. Trees, 29, 259-274.

Álvarez-Sánchez, J., Barajas-Guzmán, G., Campo, J. y León, R. (2016). Inorganic nitrogen and phosphorus in stemflow of the palm Astrocaryum mexicanum Liebm. located in Los Tuxtlas, Mexico. Tropical Ecology, 57, $45-55$.

Anaya, C. A., García-Oliva, F. y Jaramillo, V. (2007). Rainfall and labile carbon availability control litter nitrogen dynamics in a tropical dry forest. Oecologia, 150, 602-610.

Anaya, C. A., Jaramillo, J. L., Martínez-Yrízar, A. y García-Oliva, F. (2012). Large rainfall pulses control litter decomposition in a tropical dry forest: Evidence from an 8-year study. Ecosystems, 15, 652-663.

Arredondo, T., García-Moya, E., Huber-Sannwald, E., Loescher, H. W., Delgado-Balbuena, J. y Luna, M. (2016). Drought manipulation and its direct and legacy effect on productivity of a monodominant and mixed-species semiarid grassland. Agricultural and Forest Meteorology, 223, 132-140.

Ballinas, M., Esperón-Rodríguez, M. y Barradas, V. L. (2015). Estimating evapotranspiration in the central mountain region of Veracruz, Mexico. Bosque, 36, 445-455.

Balvanera, P., Arias, E., Rodríguez-Estrella, R., Almeida, L., y Schmitter, J. J. (Eds.). (2016). Una mirada al conocimiento de los ecosistemas de México. México D.F.: Conacyt/UNAM.

Barradas, V. L. y Fanjul, L. (1985). Equilibrio hídrico y evapotranspiración en una selva baja caducifolia de la costa de Jalisco, México. Biotica, 10, 199-210.

Bejarano, M., Etchevers, J., Ruíz-Suárez, G. y Campo, J. (2014). The effects of increased $\mathrm{N}$ input on soil $\mathrm{C}$ and $\mathrm{N}$ dynamics in seasonally dry tropical forests: An experimental approach. Applied Soil Ecology, 73, 105-115.

Bejarano-Castillo, M., Campo, J. y Roa-Fuentes, L. (2015). Effects of increased nitrogen availability on $\mathrm{C}$ and $\mathrm{N}$ cycles in tropical forests: A meta-analysis. Plos One, 10, e0144253.

Berry, Z. C., Gotsch, S., Holwerda, F., Muñoz-Villers, L. E. y Asbjornsen, H. (2016). Slope position influences vegetation-atmosphere interactions in a tropical montane cloud forest. Agriculture and Forest Meteorology, 221, 207-228.

Bhaskar, R., Porder, S., Balvanera, P. y Edwards, E. J. (2016). Ecological and evolutionary variation in community nitrogen use traits during tropical dry forest secondary succession. Ecology, 97, 1194-1206.

Brunel, J. P. (2009). Sources of water used by natural mesquite vegetation in a semi-arid region of northern Mexico. Hydrological Sciences Journal, 54, 375-381.

Burgos, A., Maass, J. M., Ceballos, G., Equihua, M., Jardel, E., Medellín, R. A., et al. (2007). La investigación ecológica a largo plazo (LTER) y su proyección en México. Ciencia y Desarrollo, 33, 24-31.

Campo, J., Jaramillo, V. y Maass, M. (1998). Pulses of soil phosphorus availability in a Mexican tropical dry forest: Effects of seasonality and level of wetting. Oecologia, 115, 167-172.

Campo, J., Maass, J. M., Jaramillo, V. y Martínez-Yrízar, A. (2000). Calcium, potassium and magnesium cycling in a Mexican tropical dry forest ecosystem. Biogeochemistry, 49, 21-36.

Campo, J., Maass, M., Jaramillo, V., Martínez-Yrízar, A. y Sarukhán, J. (2001). Phosphorus cycling in a Mexican tropical dry forest ecosystem. Biogeochemistry, 53, 161-179.

Campo, J., Solís, E. y Gallardo, J. F. (2012). The effects of nutrient shortage on the growth of dominant tree species in secondary tropical forests (southeastern Mexico). Journal of Tropical Forest Science, 24, 419-426.

Campo, J., Solís, E. y Valencia, M. (2007). Litter N and P dynamics in two secondary tropical dry forests after relaxation of nutrient availability constraints. Forest Ecology and Management, 252, 33-40.

Campos, A. (2010). Response of soil inorganic nitrogen to land use and topographic position in the Cofre de Perote Volcano (Mexico). Environmental Management, 46, 213-224.

Campos, A., Etchevers, J., Oleschko, K. y Hidalgo, C. (2012). Soil microbial biomass and nitrogen mineralization rates along an altitudinal gradient on the Cofre de Perote Volcano (Mexico): The importance of landscape position and land use. Land Degradation and Development., 25, 581-593.

Campos, A., Oleschko, K., Etchevers, J. y Hidalgo, C. (2007). Exploring the effect of changes in land use on soil quality on the eastern slope of the Cofre de Perote Volcano (Mexico). Forest Ecology and Management., 248, 174-182.

Cantú-Silva, I. y González-Rodríguez, H. (2001). Interception loss, throughfall and stemflow chemistry in pine and oak forests in northeastern Mexico. Tree Physiology, 21, 1009-1013.

Cárdenas, I. y Campo, J. (2007). Foliar nitrogen and phosphorus resorption and decomposition in the nitrogen-fixing tree Lysiloma microphyllum in primary and secondary seasonally tropical dry forests in Mexico. Journal of Tropical Ecology, 23, 107-113.

Carlyle-Moses, D. E., Flores Laureano, J. S. y Price, A. G. (2004). Throughfall and throughfall spatial variability in Madrean oak forest communities of northeastern Mexico. Journal of Hydrology, 297, 124-135.

Carlyle-Moses, D. E. y Price, A. G. (2007). Modelling canopy interception loss from a Madrean pine-oak stand, Northeastern Mexico. Hidrological Processes, 21, 2572-2580.

Ceccon, E., Sánchez, S. y Campo, J. (2002). Tree seedling dynamics in two abandoned tropical dry forests of differing successional status in Yucatán, Mexico: A field experiment with $\mathrm{N}$ and P fertilization. Plant Ecology, 170, 277-285.

Cervantes, L., Maass, J. M. y Domínguez, R. (1988). Relación lluviaescurrimiento en un sistema pequeño de cuencas de selva baja caducifolia. Ingeniería Hidráulica en México. Segunda Época, III, 30-42.

Challenger, A., Bocco, G., Equihua, M., Lazos-Chavero, E. y Maass, J. M. (2015). La aplicación del concepto del sistema socio-ecológico: alcances, posibilidades y limitaciones en la gestión ambiental de México. Investigación Ambiental y Política Pública, 6, 1-21.

Chávez-Bergara, B., Rosales-Castillo, A., Merino, A., Vázquez-Marrufo, G., Oyama, K. y García-Oliva, F. (2016). Quercus species control nutrients dynamics by determining the composition and activity of the forest floor fungal community. Soil Biology and Biochemistry, 98, 186-195.

Das, R., Lawrence, D., d'Odorico, P. y DeLonge, M. (2011). Impact of land use change on atmospheric P inputs in a tropical dry forest. Journal of Geophysical Research, 116, 1-9. G01027

Davidson, E. A., Matson, P. A., Vitousek, P., Riley, R., Dunkin, K., GarcíaMéndez, G., et al. (1993). Process regulation of soil emission of NO and $\mathrm{N} 2 \mathrm{O}$ in a seasonally dry tropical forest. Ecology, 74, 130-139.

Davidson, E. A., Vitousek, P., Matson, P. A., Riley, R., García-Méndez, G., Maass, J. M., et al. (1991). Soil emissions of nitric oxide in a seasonally dry tropical forest of Mexico. Journal of Geophysical Research, 96, $15439-15445$.

Escobar, E., Maass, M., Alcocer, J., Azpra, E., Falcón, L., Gallegos, A., et al. (2008). Diversidad de procesos funcionales en los ecosistemas. En J. Soberón, G. Halffter, y J. Llorente-Bousquets (Eds.), Capital Natural de México. Vol. I. Conocimiento actual de la biodiversidad (pp. 161-189). México D.F.: Conabio.

Esperón-Rodríguez, M. y Barradas, V. L. (2015). Comparing environmental vulnerability in the montane cloud forest of eastern Mexico: A vulnerability index. Ecological Indicators, 52, 300-310.

Fenn, M. E., de Bauer, L., Quevedo-Nolasco, A. y Rodríguez-Frausto, C. (1999). Nitrogen and sulfur deposition and forest nutrient status in the Valley of Mexico. Water Air and Soil Pollution, 113, 155-174.

Galicia, L. y García-Oliva, F. (2004). The effects of C, N and P addition on soil microbial activity under two remnant tree species in a tropical seasonal pasture. Applied Soil Ecology, 26, 31-39.

Galicia, L. y García-Oliva, F. (2008). Remnant tree effects on soil microbial carbon and nitrogen in tropical seasonal pastures in western Mexico. European Journal of soil Biology, 44, 290-297.

Galicia, L., López-Blanco, J., Zarco-Arista, A. E., Filips, V. y García-Oliva, F. (1999). The relationship between solar radiation interception and soil water content in a tropical deciduous forest in Mexico. Catena, 36, 153-164.

Gamboa, A. M., Hidalgo, C., de León, F., Etchevers, J., Gallardo, J. F. y Campo, J. (2010). Nutrient addition differentially affects soil carbon sequestration in secondary tropical dry forests: Early- vs. late-succession stages. Restoration Ecology, 18, 252-260. 
García-Méndez, G., Maass, J. M., Matson, P. A. y Vitousek, P. (1991). Nitrogen transformations and nitrous-oxide flux in a tropical deciduous forest in Mexico. Oecologia, 88, 362-366.

García-Oliva, F., Casar, I., Morales, P. y Maass, J. M. (1994). Forest-to-pasture conversion influences on soil carbon dynamics in a Tropical Deciduous Forest. Oecologia, 99, 392-396.

García-Oliva, F., Gallardo, J. y Montaño, N. (2006). Soil carbon and nitrogen dynamics followed by a forest-to-pasture conversion in western Mexico. Agroforestry Systems, 66, 93-100.

García-Oliva, F., Maass, J. M. y Galicia, L. (1995). Rainstorm analysis and rainfall erosivity of a seasonal tropical region with a strong cyclonic influence in the Pacific coast of Mexico. Journal of Applied Meteorology, 34, 2491-2498.

García-Oliva, F., Martínez, R. y Maass, J. M. (1995). Long-term net soil erosion as determined by Cs-137 redistribution in a natural and perturbed tropical deciduous forest ecosystem. Geoderma, 68, 135-147.

Gavito, M. E., Martínez-Yrizar, A., Ahedo, R., Araiza, S., Ayala, B., Ayala, R., et al. (2014). La vulnerabilidad del socio-ecosistema de bosque tropical seco de Chamela, Jalisco, al cambio global: un análisis de sus componentes ecológicos y sociales. Investigación Ambiental Ciencia y Política Pública, 6, 109-126.

Gebremichael, M. y Barros, A. P. (2006). Evaluation of MODIS Gross Primary Productivity (GPP) in tropical monsoon regions. Remote Sensing of Environment, 100, 150-166.

Giardina, C. P., Sanford, R. L., Jr. y Døckersmith, I. C. (2000). Changes in soil phosphorus and nitrogen during slash-and-burn clearing of a dry tropical forest. Soil Science Society of American Journal, 64, 399-405.

Goldsmith, G. R., Muñoz-Villers, L. E., Holwerda, F., Mcdonnell, J. J., Asbjornsen, H. y Dawson, T. E. (2012). Stable isotopes reveal linkages among ecohydrological processes in a seasonally dry tropical montane cloud forest. Ecohydrology, 5, 779-790.

Gómez-Tagle, C. A., Gómez-Tagle, R. A. F., Ávila, O. J. A. y Bruijnzeel, L. A. (2015). Partición de la precipitación en un bosque tropical montano de pino-encino en el centro de México. Bosque (Valdivia), 36, 505-518.

Gondwe, B. R. N., Lerer, S., Stisen, S., Marín, L., Rebolledo-Vieyra, M., Merediz-Alonso, G., et al. (2010). Hydrogeology of the south-eastern Yucatan Peninsula: New insights from water level measurements, geochemistry, geophysics and remote sensing. Journal of Hydrology, 389, 1-17.

González-Ruíz, T., Jaramillo, V. J., Peña-Cabriales, J. J. y Flores, A. (2008). Nodulation dynamics and nodule activity in leguminous tree species of a Mexican tropical dry forest. Journal of Tropical Ecology, 24, 107-110.

Hastings, S. J., Oechel, W. C. y Muhlia-Melo, A. (2005). Diurnal, seasonal and annual variation in the net ecosystem $\mathrm{CO} 2$ exchange of a desert shrub community (sarcocaulescent) in Baja California, Mexico. Global Change Biology, 11, 927-939.

Herrera-Arreola, G., Herrera, Y., Reyes-Reyes, B. G. y Dendooven, L. (2007). Mesquite (Prosopis juliflora (Sw.) DC.), huisache (Acacia farnesiana (L.) Willd.) and catclaw (Mimosa biuncifera Benth.) and their effect on dynamics of carbon and nitrogen in soils of the semi-arid highlands of Durango, Mexico. Journal of Arid Environments, 69, 583-598.

Holwerda, F., Bruijnzeel, L. A., Muñoz-Villers, L. E., Equihua, M. y Asbjornsen, H. (2010). Rainfall and cloud water interception in mature and secondary lower montane cloud forests of central Veracruz, Mexico. Journal of Hydrology, 384, 84-96.

Huber-Sannwald, E., Maestre, F. T., Herrick, J. E. y Reynolds, J. F. (2006) Ecohydrological feedbacks and linkages associated with land degradation: A case study from Mexico. Hydrological Process, 20, 3395-3411.

Hughes, R. F., Kauffman, J. y Jaramillo, V. (1999). Biomass, carbon and nutrient dynamics of secondary forest in a humid tropical region of Mexico. Ecology, 80, 1892-1907.

Hughes, R. F., Kauffman, J. y Jaramillo, V. (2000). Ecosystem-scale impacts of deforestation and land use in a humid tropical region of Mexico. Ecological Application, 10, 515-527.

Jaramillo, V., Kauffman, J. B., Rentería-Rodríguez, L., Cummings, D. L. y Ellingson, L. J. (2003). Biomass, carbon, and nitrogen pools in Mexican tropical dry forest landscapes. Ecosystems, 6, 609-629.

Jaramillo, V., Martínez-Yrízar, A. y Sanford, R. L., Jr. (2011). Primary productivity and biogeochemistry of primary and secondary tropical dry forests. En R. Dirzo, H. Young, H. A. Mooney, y G. Ceballos (Eds.), Seasonally Dry
Tropical Forests: Ecology and Conservation (pp. 109-128). Washington, D.C.: Island Press.

Jardel, E., Maass, J. M., y Rivera-Monroy, V. H. (Eds.). (2013). Investigación ecológica a largo plazo en México. Ciudad de México: Red Mexicana de Investigación Ecológica a Largo Plazo. Guadalajara: Universidad de Guadalajara.

Kellman, M. y Roulet, M. (1990). Stemflow and throughfall in a tropical dry forest. Earth Surface Processes and Landforms, 15, 55-61.

LeBauer, D. S. y Treseder, K. K. (2008). Nitrogen limitation of net primary productivity in terrestrial ecosystems is globally distributed. Ecology, 89, 371-379.

Maass, J. M. (1992). The use of litter-mulch to reduce erosion on hilly land in Mexico. En H. Hurni y K. Tato (Eds.), Erosion, Conservation and SmallScale Farming (pp. 383-391). Berna: Geographisca Bernensia, International Soil. Conservation Organization (ISCO) and World Ass. of Soil and Water Conservation (WASWC)

Maass, J. M. (1995). Tropical deciduous forest conversion to pasture and agriculture. En S. H. Bullock, H. A. Mooney, y E. Medina (Eds.), Seasonally Dry Tropical Forests (pp. 399-422). Cambridge: Cambridge University Press.

Maass, J. M. y Burgos, A. (2011). Water dynamics at the ecosystem level in tropical dry forests. En R. Dirzo, H. Young, H. A. Mooney, y G. Ceballos (Eds.), Seasonally Dry Tropical Forests: Ecology and Conservation (pp. 141-156). Washington, D.C.: Island Press.

Maass, J. M., Jaramillo, V., Martínez-Yrízar, A., García-Oliva, F., PérezJiménez, A. y Sarukhán, J. (2002). Aspectos funcionales del ecosistema de selva baja caducifolia en Chamela, Jalisco. En F. A. Noguera, J. H. Vega-Rivera, A. N. García-Aldrete, y M. Quezada-Avendaño (Eds.), Historia Natural de Chamela (pp. 525-542). Ciudad de México: Instituto de Biología, UNAM.

Maass, J. M., Jordan, C. y Sarukhán, J. (1988). Soil erosion and nutrient losses in seasonal tropical agroecosystems under various management techniques. Journal of Applied Ecology, 25, 595-607.

Maass, M., Díaz-Delgado, R., Balvanera, P., Castillo, A. y Martínez-Yrízar, A. (2010). Redes de investigación ecológica y socio-ecológica a largo plazo (LTER y LTSER) en Iberoamérica: los casos de México y España. Revista Chilena de Historia Natural, 83, 171-184.

Maass, M., Equihua, M. y Jardel, E. (2008). La Red Mexicana de Investigación Ecológica a Largo Plazo. Editores invitados para el número especial. Ciencia y Desarrollo, 34, 1-215.

Medina-Roldán, E., Arredondo, T., Huber-Sannwald, E., Chapa-Vargas, L. y Olalde, V. (2008). Grazing effects on fungal root symbionts and carbon and nitrogen storage in a shortgrass steppe in Central Mexico. Journal of Arid Environments, 72, 546-556.

Méndez-Barroso, L. A. y Vivoni, E. R. (2010). Observed shifts in land surface conditions during the North American Monsoon: Implications for a vegetation-rainfall feedback mechanism. Journal of Arid Environments, 74, $549-555$.

Méndez-Barroso, L. A., Vivoni, E. R., Robles-Morua, A., Mascaro, G., Yépez, E. A., Rodríguez, J. C., et al. (2014). A modeling approach reveals differences in evapotranspiration and its partitioning in two semiarid ecosystems in Northwest Mexico. Water Resources Research, 50, 3229-3252.

Méndez-Barroso, L. A., Vivoni, E. R., Watts, C. J. y Rodríguez, J. C. (2009). Seasonal and interannual relations between precipitation, surface soil moisture and vegetation dynamics in the North American monsoon region. Journal of Hydrology, 377, 59-70.

Millennium Ecosystem Assessment. (2005). Ecosystems and Human WellBeing: Synthesis. Washington, D.C.: Island Press.

Montaño, N., García-Oliva, F. y Jaramillo, V. (2007). Dissolved organic carbon affects soil microbial activity and nitrogen dynamics in a Mexican tropical deciduous forest. Plant and Soil, 295, 265-277.

Mora, F. y Iverson, L. R. (1998). On the sources of vegetation activity variation, and their relation with water balance in Mexico. International Journal of Remote Sensing, 19, 1843-1871.

Morales-Romero, D., Campo, J., Godínez-Álvarez, H. y Molina-Freaner, F. (2015). Soil carbon, nitrogen and phosphorus change from conversions of thornscrub to buffelgrass pasture in northwestern Mexico. Agriculture, Ecosystems and Environment, 199, 231-237. 
Muñoz-Villers, L. E., Geissert, D. R., Holwerda, F. y McDonnell, J. J. (2016). Factors influencing stream baseflow transit times in tropical montane watersheds. Hydrology and Earth Systems Science, 20, $1621-1635$

Muñoz-Villers, L. E., Holwerda, F., Alvarado-Barrientos, M. S., Geissert, D., Marín-Castro, B. E., Gómez-Tagle, A., et al. (2015). Efectos hidrológicos de la conversión del bosque de niebla en el centro de Veracruz, México. Bosque, 36, 395-407.

Muñoz-Villers, L. E., Holwerda, F., Gómez-Cárdenas, M., Equihua, M., Asbjornsen, H., Bruijnzeel, L. A., et al. (2012). Water balances of old-growth and regenerating montane cloud forests in central Veracruz, Mexico. Journal of Hydrology, 462-463, 53-66.

Muñoz-Villers, L. E. y McDonnell, J. J. (2013). Land use change effects on runoff generation in a humid tropical montane cloud forest region. Hydrology and Earth System Sciences, 17, 3543-3560.

Návar, J. (1993). The causes of stemflow variation in three semi-arid growing species of northeastern Mexico. Journal of Hydrology, 145, 179-190.

Návar, J. (2011). Stemflow variation in Mexico's northeastern forest communities: Its contribution to soil moisture content and aquifer recharge. Journal of Hydrology, 408, 35-42.

Návar, J. (2013). The performance of the reformulated Gash's interception loss model in Mexico's northeastern temperate forests. Hydrological Processes, $27,1626-1633$.

Návar, J. (2015). Hydro-climatic variability and perturbations in Mexico's northwestern temperate forests. Ecohydrology, 8, 1065-1072.

Návar, J. y Bryan, R. (1990). Interception loss and rainfall redistribution by three semi-arid growing shrubs in northeastern Mexico. Journal of Hydrology, $115,51-63$

Návar, J., Carlyle-Moses, D. E. y Martínez, M. A. (1999). Interception loss from the Tamaulipan matorral thornscrub of north-eastern Mexico: An application of the Gash analytical interception loss model. Journal of Arid Environments, $41,1-10$.

Návar, J., Charles, F. y Jurado, E. (1999). Spatial variations of interception loss components by Tamaulipan thornscrub in northeastern Mexico. Forest Ecology and Management, 124, 231-239.

Noguez, A. M., Escalante, A. E., Forney, L. J., Nava-Mendoza, M., Rosas, I., Souza, V., et al. (2008). Soil aggregates in a tropical deciduous forest: Effects on $\mathrm{C}$ and $\mathrm{N}$ dynamics, and microbial communities as determined by t-RFLPs. Biogeochemistry, 89, 209-220.

Pérez-Ruíz, E. R., Garatuza-Payán, J., Watts, C. J., Rodríguez, J. C., Yepez, E. A. y Scott, R. L. (2010). Carbon dioxide and water vapour exchange in a tropical dry forest as influenced by the North American Monsoon System (NAMS). Journal of Arid Environments, 74, 556-563.

Pérez-Suárez, M., Arredondo-Moreno, J. T., Huber-Sannwald, E. y Serna-Pérez, A. (2014). Forest structure, species traits and rain characteristics influences on horizontal and vertical rainfall partitioning in a semiarid pine-oak forest from Central Mexico. Ecohydrology, 7, 532-543.

Pérez-Suárez, M., Fenn, M. E., Cetina-Alcalá, V. M. y Aldrete, A. (2008). The effects of canopy cover on throughfall and soil chemistry in two forest sites in the Mexico City air basin. Atmosfera, 21, 83-100.

Perroni-Ventura, Y., Montaña, C. y García-Oliva, F. (2006). Relationship between soil nutrient availability and plant species richness in a tropical semi-arid environment. Journal of Vegetation Science, 17, 719-728.

Ponette-González, A. G., Weathers, K. C. y Curran, L. M. (2010). Water inputs across a tropical montane landscape in Veracruz, Mexico: Synergistic effects of land cover, rain and fog seasonality, and interannual precipitation variability. Global Change Biology, 16, 946-963.

Porporato, A. y Rodríguez-Iturbide, I. (2002). Ecohydrology — a challenging multidisciplinary research perspective /Ecohydrologie: une perspective stimulante de recherche multidisciplinaire. Hydrological Sciences Journal, 47, 811-821.

Portillo-Quintero, C. A. y Sánchez-Azofeifa, G. A. (2010). Extent and conservation of tropical deciduous forests in America. Biological Conservation, 143, 144-155.

Rentería, L. y Jaramillo, V. (2011). Rainfall drives leaf traits and leaf nutrient resorption in a tropical dry forest in Mexico. Oecologia, 165, 201-211.
Rentería, L., Jaramillo, V., Martínez-Yrízar, A. y Pérez-Jiménez, A. (2005). Nitrogen and phosphorus resorption in trees of a Mexican tropical dry forest. Trees, 19, 431-441.

Reyes-Gómez, V. M., Viramontes-Pereida, D., Miranda-Ojeda, N. E., SánchezFernández, P. B. y Viramontes-Olivas, O. (2007). Papel hidrológico ambiental de las propiedades hidráulicas del suelo superficial de la cuenca del Río Conchos. Ingeniería Hidráulica en México, 22, 33-46.

Riensche, M., Castillo, A., Flores-Díaz, A. y Maass, M. (2015). Tourism at Costalegre, Mexico: An ecosystem services-based exploration of current challenges and alternative futures. Futures, 66, 70-84.

Rivera-Monroy, V., Maass, M., Benítez, J., Coronado, C., Euán, J., Godínez, E., et al. (2008). Eco-hidrología y demandas de agua en México. Ciencia y Desarrollo, 34, 24-27.

Robles-Morua, A., Vivoni, E. R. y Mayer, A. S. (2012). Distributed hydrologic modeling in northwest Mexico reveals the links between runoff mechanisms and evapotranspiration. Journal of Hydrometeorology, 13, 785-807.

Runyan, C., d'Odorico, P., Vandecar, K., Das, R., Schmoock, B. y Lawrence, D. (2013). Positive feedbacks between phosphorus deposition and forest canopy trapping, evidence from Southern Mexico. Journal of Geophysical Research, 118, 1521-1531.

Rzedowski, J. (2006). Vegetación de México (Primera edición digital). Ciudad de México: Comisión Nacional para el Conocimiento y Uso de la Biodiversidad.

Sandoval-Pérez, A., Camargo-Ricalde, S. L., Montaño, N., García-Oliva, F., Alarcón, A., Montaño, S., et al. (2016). Biocrust, inside and outside resource islands of Mimosa luisana (Leguminosae), improve soil carbon and nitrogen dynamics in a tropical semiarid ecosystem. European Journal of Soil Biology, 74, 93-103.

Sarukhán, J. y Maass, J. M. (1990). Bases ecológicas para un manejo sostenido de los ecosistemas: el sistema de cuencas hidrológicas. En E. Leff (Ed.), Medio ambiente y desarrollo en México. Vol. I (pp. 81-114). Ciudad de México: UNAM (CIIH)-Porrúa.

Saynes, V., Hidalgo, C., Etchevers, J. y Campo, J. (2005). Soil C and N dynamics in primary and secondary seasonally dry tropical forests in Mexico. Applied Soil Ecology, 29, 282-289.

Tang, Q., Vivoni, E. R., Muñoz-Arriola, F. y Lettenmaier, D. P. (2012). Predictability of evapotranspiration patterns using remotely sensed vegetation dynamics during the North American Monsoon. Journal of Hydrometeorology, 13, 103-121.

Tapia-Torres, Y., López-Lozano, N. E., Souza, V. y García-Oliva, F. (2015) Vegetation-soil system controls soil mechanisms for nitrogen transformations in an oligotrophic Mexican desert. Journal of Arid Environments, 114, $62-69$.

Tarin, T., Yépez, E. A., Garatuza-Payán, J., Watts, C. J., Rodríguez, J. C., Vivoni, E. R., et al. (2014). Partición de la evapotranspiración usando isótopos estables en estudios ecohidrológicos. Tecnología y Ciencias del Agua, 5, 97-114.

Tobón, W., Martínez-Garza, C. y Campo, J. (2010). Soil responses to restoration of a tropical pasture in Veracruz, South-Eastern Mexico. Journal of Tropical Forest Science, 23, 338-344.

Toledo-Aceves, T. y García-Oliva, F. (2008). Effects of forest-pasture edges on $\mathrm{C}, \mathrm{N}$ and $\mathrm{P}$ associated with Caesalpinia eriostachys, a dominant tree species in a tropical deciduous forest in Mexico. Ecological Research, 23, 271-280.

Valdespino, P., Romualdo, R., Cardenazzi, L. y Campo, J. (2009). Phosphorus cycling in primary and secondary seasonally dry tropical forests in Mexico. Annals of Forest Science, 66, 107-107

Vargas, R., Loescher, H. W., Arredondo, T., Huber-Sannwald, E., Lara-Lara, R. y Yépez, E. A. (2012). Opportunities for advancing carbon cycle science in Mexico: Toward a continental scale understanding. Environmental Science and Policy, 21, 84-93.

Vargas, R., Yépez, A., Andrade, J. L., Ángeles, G., Arredondo, T., Castellanos, A. E., et al. (2013). Progress and opportunities for monitoring greenhouse gases fluxes in Mexican ecosystems: The MexFlux network. Atmósfera, 26, 325-336.

Verduzco, V. S., Garatuza-Payán, J., Yépez, E. A., Watts, C. J., Rodríguez, J. C., Robles-Morua, A., et al. (2015). Variations of net ecosystem production due to seasonal precipitation differences in a tropical dry forest of 
northwest Mexico. Journal of Geophysical Research G: Biogeosciences, 120, 2081-2094.

Villarreal, S., Vargas, R., Yepez, E. A., Acosta, J. S., Castro, A., EscotoRodriguez, M., et al. (2016). Contrasting precipitation seasonality influences evapotranspiration dynamics in water-limited shrublands. Journal of Geophysical Research G: Biogeosciences, 121, 494-508.

Vitousek, P., Matson, P., Volkmann, C., Maass, J. M. y García, G. (1989). Nitrous oxide flux from dry tropical forests. Global Biogeochemical Cycles, 3, 375-382.

Vivoni, E. R. (2012). Diagnosing seasonal vegetation impacts on evapotranspiration and its partitioning at the catchment scale during SMEX04-NAME. Journal of Hydrometeorology, 13, 1631-1638.
Vivoni, E. R., Watts, C. J., Rodríguez, J. C., Garatuza-Payán, J., MéndezBarroso, L. A. y Sáiz-Hernández, J. A. (2010). Improved land-atmosphere relations through distributed footprint sampling in a subtropical scrubland during the North American monsoon. Journal of Arid Environments, 74, $579-584$.

Vose, J. M. y Maass, J. M. (1999). A comparative analysis of hydrologic responses of tropical deciduous and temperate deciduous watershed ecosystems to climatic change. En C. Aguirre-Bravo y C. RodríguezFranco (Eds.), North American Science Symposium: Toward a unified framework for inventorying and monitoring forest ecosystem resources (pp. 292-298). Guadalajara, México: USDA Forest Service Proceedings RMRS. 